\title{
A spectrophotometric atlas of Narrow-Line Seyfert 1 galaxies
}

\author{
M.-P. Véron-Cetty ${ }^{1}$, P. Véron ${ }^{1}$, and A. C. Gonçalves ${ }^{2}$ \\ 1 Observatoire de Haute Provence, CNRS, 04870 Saint-Michel l'Observatoire, France \\ e-mail: mira@obs-hp.fr \\ 2 European Southern Observatory (ESO), Karl Schwarzschild Strasse 2, 85748 Garching bei München, Germany \\ e-mail: adarbon@eso.org
}

Received 8 February 2001 / Accepted 20 March 2001

\begin{abstract}
We have compiled a list of 83 objects classified as Narrow-Line Seyfert 1 galaxies (NLS1s) or known to have a broad Balmer component narrower than $2000 \mathrm{~km} \mathrm{~s}^{-1}$. Of these, 19 turned out to have been spectroscopically misidentified in previous studies; only 64 of the selected objects are genuine NLS1s. We have spectroscopically observed 59 of them and tried to characterize their Narrow and Broad-Line Regions (NLR and BLR) by fitting the emission-lines with Gaussian and/or Lorentzian profiles.

In most cases, the broad Balmer components are well fitted by a single Lorentzian profile, confirming previous claims that Lorentzian rather than Gaussian profiles are better suited to reproduce the shape of the NLS1s broad emission lines. This has consequences concerning their FWHMs and line ratios: when the broad Balmer components are fitted with a Lorentzian, most narrow line regions have line ratios typical of Seyfert 2s while, when a Gaussian profile is used for fitting the broad Balmer components, the line ratios are widely scattered in the usual diagnostic diagrams (Veilleux \& Osterbrock 1987); moreover, the FWHM of the best fitting Lorentzian is systematically smaller than the FWHM of the Gaussian.

We find that, in general, the [O III] lines have a relatively narrow Gaussian profile $\left(\sim 200-500 \mathrm{~km} \mathrm{~s}^{-1} F W H M\right)$ with often, in addition, a second broad ( $\left.\sim 500-1800 \mathrm{~km} \mathrm{~s}^{-1} F W H M\right)$, blueshifted Gaussian component. We do not confirm that the [O III] lines are weak in NLS1s.

As previously suggested, there is a continuous transition of all properties between NLS1s and classical Broad-Line Seyfert 1 Galaxies (BLS1s) and the limit of $2000 \mathrm{~km} \mathrm{~s}^{-1}$ used to separate the two species is arbitrary; $R_{4570}$, the ratio of the $\mathrm{Fe}$ II to the $\mathrm{H} \beta$ fluxes, could be a physically more meaningful parameter to distinguish them.
\end{abstract}

Key words. galaxies: Seyfert

\section{Introduction}

Osterbrock \& Pogge (1985) have identified a class of AGNs having all properties of the Seyfert 1s with, however, very narrow Balmer lines and strong optical Fe II lines; they are called NLS1s. Quantitatively, a Seyfert 1 is called an NLS1 if the "broad" component of the Balmer lines is narrower than $2000 \mathrm{~km} \mathrm{~s}^{-1} F W H M$ (Osterbrock 1987). NLS1s often have strong Fe II emission; many of them have a strong soft X-ray excess and display high-amplitude $\mathrm{X}$-ray variability.

The 2-10 keV spectrum of classical BLS1s can be fitted with a power-law with a photon index $\Gamma=1.73 \pm 0.05$ (Nandra \& Pounds 1994; Reynolds 1997; George et al. 1998a); in NLS1s, $\Gamma$ is significantly steeper $(\Gamma=2.19 \pm$ 0.10) (Leighly 1999b); in fact, it is anticorrelated with the H $\beta$ FWHM (Brandt et al. 1997; Reeves \& Turner 2000).

Some Seyfert 1s show evidence for an excess of soft Xrays above the hard $\mathrm{X}$-ray power-law extrapolation, dominant below $\sim 1 \mathrm{keV}$ (Saxton et al. 1993; George et al. 2000). This excess is more important and more frequent

Send offprint requests to: P. Véron, e-mail: veron@obs-hp.fr in NLS1s than in BLS1s (Vaughan et al. 1999a; Leighly 1999b; Reeves \& Turner 2000). The soft photon spectral index $\Gamma(0.1-2.4 \mathrm{keV})$, which measures the relative strength of the soft component, is correlated with the Balmer line width in the sense that steep soft X-ray spectra corresponds to narrow Balmer lines (Puchnarewicz et al. 1992; Boller et al. 1996; Wang et al. 1996; Laor et al. 1997a). In fact NLS1s may show both steep as well as flat X-ray spectra, while BLS1s always have flat spectra (Grupe et al. 1999). However, several NLS1s show a significant intrinsic neutral hydrogen column density in excess of the Galactic value; the unability to detect a soft excess in the X-ray spectrum of some NLS1s could be due to the presence of such a high column density. Therefore the soft excess may be more prevalent in NLS1s than observed (Leighly 1999b).

Most AGN spectra show the presence of a "Big Blue Bump" (BBB) extending from optical frequencies upwards (Elvis et al. 1986; Sanders et al. 1989). The BBB has been interpreted as the thermal emission of a physically thin, optically thick accretion disk (Sun \& Malkan 1989; Siemiginowska et al. 1995). The soft X-ray spectral index 
is correlated to the strength of the ultraviolet bump in unabsorbed Seyfert 1s, indicating that the BBB is in fact an ultraviolet to soft X-ray bump (Walter \& Fink 1993; Puchnarewicz et al. 1995; Page et al. 1999). NLS1s have significantly bluer spectra than BLS1s which is consistent with the presence of a more pronounced BBB in NLS1s (Grupe et al. 1998). However, when sufficient data are available, it seems that a single standard accretion disk model cannot fit the optical/UV/X-ray bump (Kolman et al. 1993; Wisotzki et al. 1995).

NLS1s very frequently exhibit rapid and/or highamplitude X-ray variability (Boller et al. 1996; Forster \& Halpern 1996; Molthagen et al. 1998). The X-ray-steep, narrow-H $\beta$ AGNs systematically show larger amplitude variations than the X-ray-flat, broad-H $\beta$ AGNs on time scales from 2 to 20 days (Fiore et al. 1998; Leighly 1999a; Turner et al. 1999b). Observed variability by a factor 2 in a few hours or less shows that a substantial fraction of the soft component comes from a compact region, smaller than a light-day. Giant-amplitude X-ray variability (from one up to more than two orders of magnitude on a time scale of one to a few years) has been observed in several NLS1s (see for instance Brandt et al. 1999 and Uttley et al. 1999). In the cases of NGC 4051 and IRAS 13224-3809, changes by a factor of 10 or more have occured within a few hours (Leighly 1999a). It is quite remarkable that all these objects, except NGC 4051, have a very high soft photon index $(\Gamma>4)$, at least when they are bright.

A few BLS1s have also displayed X-ray flux variability by a factor of 10 or more such as NGC 3227 $(\times 15)$ (Komossa \& Fink 1997a; George et al. 1998b) and NGC $3786(\times 10)$ (Komossa \& Fink 1997b); in both cases, however, the variability has been attributed to a change in the column density of a warm absorber.

A number of observations suggests that NLS1s are Seyfert $1 \mathrm{~s}$ with a near- or super-Eddington accretion rate.

If the broad-line emitting region is gravitationally linked to the central black hole $(\mathrm{BH})$, one can show that the FWHM of the lines depends on the mass of the $\mathrm{BH}$, the ratio of the luminosity to the Eddington luminosity and the angle between the rotation axis of the gas disk and the line of sight. The NLS1s could be either normal Seyfert 1s seen perpendicularly to the disk, or objects with a low mass BH radiating near the Eddington limit (Wang et al. 1996).

The combination of strong soft X-ray excess and steep power law prompted Pounds et al. (1995) to postulate that NLS1s represent the supermassive $\mathrm{BH}$ analogue of Galactic BH candidates (GBHC) in their high states. The high states of GBHCs are thought to be triggered by increases in the accretion rate resulting in strong thermal emission from a disk accreting at the Eddington limit.

Standard accretion disks are not able to account for the soft X-ray excess unless the Eddington ratio is close to unity; a large accretion rate results in a more pronounced $\mathrm{BBB}$ which is shifted toward higher energies, resulting in stronger soft X-ray emission and hence steeper soft X-ray slope (Pounds et al. 1987; Ross et al. 1992; Kuraszkiewicz et al. 2000).

At a fixed luminosity, BHs radiating at higher fractions of the Eddington rate will have lower masses; lower mass $\mathrm{BHs}$ are thought to be associated with physically smaller emission regions that vary more rapidly. This may explain why higher amplitude short term X-ray variability is observed in NLS1s (Fiore et al. 1998; Leighly 1999a; Turner et al. 1999b).

Nicastro (2000) proposed a model in which, for accretion rates $\dot{M} / \dot{M}_{\text {Edd }}<0.2$ (sub-Eddington regime), the predicted FWHMs are quite broad $\left(>4000 \mathrm{~km} \mathrm{~s}^{-1}\right)$, while for $\dot{M} / \dot{M}_{\mathrm{Edd}}=0.2-3$ (Eddington to moderately super-Eddington), the corresponding FWHMs span the interval $\approx 1000-4000 \mathrm{~km} \mathrm{~s}^{-1}$.

The amount of published data on NLS1s increased dramatically over the last few years, specially since the launch of the ROSAT and ASCA satellites, and important progress has been made in the X-ray domain. NLS1s, however, are still subject to much debate; our knowledge of the basic properties concerning their emission-line regions (line profiles, line ratios, etc.), and of their relation to the $\mathrm{X}$-ray properties, is still rather limited, as is the relationship between NLS1s and classical BLS1s. This is largely due to the fact that little effort has been put into providing a set of high-quality optical spectroscopic data. Published data are very heterogeneous; spectra often have a resolution insufficient to separate unambiguously the broad and narrow components of the Balmer lines; in addition, the presence of strong Fe II lines makes it difficult to measure $\mathrm{H} \beta$.

Aware of the fact that a detailed and consistent study of NLS1 emission-line properties was missing and that the knowledge of these properties is of crucial importance for understanding the basic physical differences between BLS1s and NLS1s and, ultimately, for fitting them into the standard unifying picture, we have obtained a homogeneous set of moderate resolution $(3.4 \AA F W H M$ or $200 \mathrm{~km} \mathrm{~s}^{-1}$ at $\mathrm{H} \beta$ ) spectra around $\mathrm{H} \alpha$ and/or $\mathrm{H} \beta$ of a large number of NLS1s; this setting turned out to be adequate as narrow line individual components are, in most cases, resolved with this resolution; however, in the few objects where a H II region is present near the galaxy nucleus, a better resolution would of course allow us to separate more easily the Seyfert 2 emission lines from the much narrower H II lines.

\section{Observations and data reduction}

We have compiled all 83 objects known to us before January 1998 to either to be NLS1s or to have a "broad" Balmer component narrower than $2000 \mathrm{~km} \mathrm{~s}^{-1}$, north of $\delta=-25^{\circ}$, brighter than $B=17.0$ and with $z<0.100$. We have spectroscopically observed 76 of them.

The observations were carried out during several observing runs with the spectrograph CARELEC (Lemaittre et al. 1989) attached to the Cassegrain focus of the 
Table 1. Observations dates and standard stars.

\begin{tabular}{|c|c|c|}
\hline Date & $\lambda$ range $(\AA)$ & Standard stars \\
\hline 21.03 .95 & $6500-7400$ & $\mathrm{BD} 26^{\circ} 2606$ \\
\hline 31.08 .95 & $4855-5755$ & Feige 15, BD $25^{\circ} 3941$ \\
\hline 01.09 .95 & $4855-5755$ & and $\mathrm{BD} 28^{\circ} 4211$ \\
\hline 10.05 .96 & $6700-7600$ & $\mathrm{GD} 140, \mathrm{BD} 26^{\circ} 2606$ \\
\hline 11.05 .96 & $4860-5760$ & Feige 98, Kopff 27 \\
\hline $15-16.07 .96$ & $4675-5575$ & $\mathrm{BD} 28^{\circ} 4211$ \\
\hline 24.07 .96 & $6335-7235$ & $\mathrm{BD} 28^{\circ} 4211$ \\
\hline 07.01 .97 & $4720-5620$ & EG 247 \\
\hline $09-10.01 .97$ & $6175-7075$ & EG 247 \\
\hline $04-07.03 .97$ & $4825-5725$ & Feige 66 \\
\hline $08-12.03 .97$ & $6310-7210$ & Feige 66 \\
\hline 13.03 .97 & $4825-5725$ & Feige 66 \\
\hline 29.10 .97 & $6500-6950$ & Feige 24, EG 247 \\
\hline 30.10 .97 & $4825-5280$ & Feige 24, EG 247 \\
\hline 31.10 .97 & $6455-7355$ & Feige 24 \\
\hline $01-02.11 .97$ & $4655-5555$ & Feige 24 \\
\hline $27-29.05 .98$ & $4645-5545$ & Feige 66, Kopff 27 \\
\hline 31.05 .98 & $6430-7330$ & Feige 66 \\
\hline 15.06 .98 & $4420-6265$ & GD 190 \\
\hline 16.06 .98 & $6020-7870$ & GD 190 \\
\hline 23.09 .00 & $4255-6090$ & Feige 15, EG 247 \\
\hline
\end{tabular}

- On October 29 and 30, 1997, we have used a dispersion of $33 \AA \mathrm{mm}^{-1}$ instead of $66 \AA \mathrm{mm}^{-1}$.

- In June 1998 and September 2000, we have used an EEV 42-20 instead of a TK 512 CCD.

Observatoire de Haute-Provence (OHP) $1.93 \mathrm{~m}$ telescope. Table 1 gives the list of observing runs with the observed wavelength ranges and the standard stars used.

The detector was a $512 \times 512$ pixels, $27 \times 27 \mu \mathrm{m}$ Tektronic CCD, except in June 1998 and September 2000 when we used a $1024 \times 2048$ pixels, $13.5 \times 13.5 \mu \mathrm{m} \mathrm{EEV}$ 42-20 CCD. We generally used a $600 \mathrm{lmm}^{-1}$ grating resulting in a dispersion of $66 \AA \mathrm{mm}^{-1}$. On October 29 and 30, 1997, we used a dispersion of $33 \AA \mathrm{mm}^{-1}$. In each case, the galaxy nucleus was centered on the slit. Three to five columns of the CCD $\left(3\right.$ to $\left.5^{\prime \prime}\right)$ were extracted on the Tektronic CCD and 7 column $\left(\sim 3^{\prime \prime}\right)$ on the EEV. The slit width was 2 .' 1 , corresponding to a projected slit width on the detector of $52 \mu \mathrm{m}$ i.e. 1.9 and 3.8 pixels with the Tektronic and the EEV CCD respectively. The resolution, as measured on the night sky lines, was $\sim 3.4 \AA F W H M$.

The spectra were flux calibrated using the standard stars given in Table 1, taken from Oke (1974); Stone (1977); Oke \& Gunn (1983) and Massey et al. (1988).

The spectra were analysed as described in Véron et al. (1980; 1981a,b) and Gonçalves et al. (1999a). Briefly, the three emission lines, $\mathrm{H} \alpha$ and $[\mathrm{N} \mathrm{II}] \lambda \lambda 6548,6583$ (or $\mathrm{H} \beta$ and $[\mathrm{O}$ III $] \lambda \lambda 4959,5007)$ were fitted by one or several sets of three Gaussian components; the width and redshift of each component in a set were forced to be the same and the intensity ratios of the $[\mathrm{N}$ II] and [O III] lines were taken to be equal to their theoretical values. The broad Balmer components were fitted by one or several Gaussian or Lorentzian profiles.

Nineteen galaxies turned out to have been misidentified as NLS1s; their spectra will be published in VéronCetty et al. (2001). The 64 others are listed in Table 2.

Before analysing our blue spectra, the Fe II multiplets were removed following the method described in Boroson \& Green (1992). This consists of subtracting a suitable fraction of a Fe II template from the NLS1 spectrum so that the flux and width of the $\mathrm{H} \beta$ and [O III] lines are no longer affected by the underlying multiplet emission. Such a template is usually obtained by taking a high signal-tonoise spectrum of I Zw 1, an NLS1 showing strong narrow Fe II emission, from which the $\mathrm{H} \beta$ and [O III] lines are carefully removed. We have observed I Zw 1 with the same instrumental setting as the rest of the galaxies in our sample and used it to build an Fe II template, following this method.

Our spectra are shown in Figs. 1 to 5.

The Fe II strength is usually quantified by $R_{4570}=$ Fe II $\lambda 4570 / \mathrm{H} \beta$, i.e. the ratio of the fluxes of the $\lambda 4570 \AA$ blend measured between $\lambda 4434$ and $\lambda 4684$ and of $\mathrm{H} \beta$, including the narrow component (Boroson \& Green 1992).

We have measured the $\mathrm{H} \beta$ equivalent width $(E W)$ and the ratio $R_{4570}$ for all objects in our sample. Figure 6 shows plots of our measurements vs. published ones for the $\mathrm{H} \beta E W$ (a) and $R_{4570}(\mathrm{~b})$. Our values of the $\mathrm{H} \beta E W$ are in good agreement with those of Boroson \& Green (1992); the ratios for the 13 objects in common have a mean value of 1.0 with a dispersion of $12 \%$. Our values are also in good agreement with those of Goodrich (1989) with however a larger dispersion (40\%); this could be due partly to variability. Our measurements of $R_{4570}$ are $25 \%$ lower than the values published by Boroson \& Green (1992) (13 objects in common) and 30\% lower than those of Goodrich (1989) (10 objects); in both cases the dispersion is $\sim 25 \%$. A significant difference exists for NGC 4051.

\section{Results and discussion}

\subsection{The broad line region}

\subsubsection{High- and low-ionization lines}

In Seyfert 1s, the broad emission lines can be separated into two distinct systems: the "high-ionization lines" (HILs): C IV $\lambda 1550$, He II $\lambda 4686$, He II $\lambda 1640$, etc, and the "low-ionization lines" (LILs): Fe II, Mg II $\lambda 2800$, etc. (Collin-Souffrin \& Lasota 1988; Gaskell 2000).

The HILs and LILs show strong kinematic differences (Sulentic et al. 1995). C IV is systematically broader than Mg II (Mathews \& Wampler 1985) or $\mathrm{H} \beta$ (Wang et al. 1998) and shows a strongly blueshifted and blue asymmetric profile (Marziani et al. 1996), and He II $\lambda 4686$ systematically broader than $\mathrm{H} \beta$ (Boroson \& Green 1992; Peterson et al. 2000). The widths of $\mathrm{H} \beta$ and Fe II 
Table 2. List of NLS1s with $z<0.100, B<17.0$ and $\delta>-25^{\circ}$. Column 1: name, Col. 2: short position, Col. 3: the Galactic hydrogen column density in units of $10^{20} \mathrm{~cm}^{-2}$ Col. 4: redshift, Col. 5: B magnitude, Cols. 6 and $7: F W H M$ (in km s $\mathrm{s}^{-1}$ ) of the broad component of the Balmer lines, and reference, Cols. 8 and 9: ROSAT (0.1-2.4 keV) photon index $\Gamma$ resulting from a power-law fit with Galactic absorption, and reference, Col. 10: ROSAT X-ray flux in cts s ${ }^{-1}$ in the energy band 0.1-2.4 keV either from the RASS catalogue or from the 1WGA catalogue $(*)$, Col. 11: "A" if we have obtained a red spectrum, "B" for a blue spectrum. References: (1) Appenzeller \& Wagner (1991); (2) Bade et al. (1995); (3) Bassani et al. (1989); (4) Boller et al. (1992); (5) Boller et al. (1996); (6) Boroson \& Green (1992); (7) Boroson \& Meyers (1992); (8) Brandt et al. (1995); (9) Ciliegi \& Maccacaro (1996); (10) Goodrich (1989); (11) Green et al. (1989); (12) Grupe et al. (1998); (13) Grupe et al. (1999); (14) Leighly (1999b); (15) Maza \& Ruiz (1989); (16) Moran et al. (1996); (17) Netzer et al. (1987); (18) Osterbrock (1977a); (19) Osterbrock \& de Robertis (1985); (20) Osterbrock \& Pogge (1985); (21) Osterbrock \& Pogge (1987); (22) Osterbrock \& Shuder (1982); (23) Puchnarewicz et al. (1994); (24) Puchnarewicz et al. (1995); (25) Rush et al. (1996); (26) Stephens (1989); (27) Stirpe (1990); (28) Walter \& Fink (1993); (29) Wang et al. (1996); (30) Winkler (1992); (31) Yuan et al. (1998); (32) Zamorano et al. (1992); (33) Pfefferkorn et al. (2001).

\begin{tabular}{|c|c|c|c|c|c|c|c|c|c|c|}
\hline Name & Position & $N_{\mathrm{H}}$ & $z$ & $B$ & $F W$ & & $\Gamma$ & & $\mathrm{X}$ & \\
\hline Mark 335 & $0003+19$ & 3.8 & 0.025 & 13.7 & 1640 & (6) & $3.10 \pm 0.05$ & $(14)$ & 2.48 & $\overline{\mathrm{AB}}$ \\
\hline I Zw 1 & $0050+12$ & 5.1 & 0.061 & 14.0 & 1240 & (6) & $3.09 \pm 0.16$ & (14) & 0.82 & $\mathrm{AB}$ \\
\hline Ton S180 & $0054-22$ & 1.5 & 0.062 & 14.4 & 1000 & $(30)$ & $3.04 \pm 0.01$ & (14) & 2.53 & $\mathrm{AB}$ \\
\hline Mark 359 & $0124+18$ & 4.8 & 0.017 & 14.2 & 480 & $(22)$ & $2.4 \pm 0.1$ & (5) & 0.61 & $\mathrm{AB}$ \\
\hline MS 01442-0055 & $0144-00$ & 2.8 & 0.080 & 15.6 & 1940 & $(26)$ & $2.7 \pm 0.2$ & (5) & 0.14 & $\mathrm{AB}$ \\
\hline Mark 1044 & $0227-09$ & 3.0 & 0.016 & 14.3 & 1280 & (10) & $3.08 \pm 0.09$ & $(33)$ & 2.14 & $\mathrm{AB}$ \\
\hline HS $0328+0528$ & $0328+05$ & 8.9 & 0.046 & 16.7 & & & & & 0.22 & $\mathrm{AB}$ \\
\hline IRAS $03450+0055$ & $0345+00$ & 11.1 & 0.031 & 16.0 & 1310 & (7) & & & $* 0.06$ & - \\
\hline IRAS $04312+4008$ & $0431+40$ & 34.5 & 0.020 & 15.2 & 690 & (16) & $2.8 \pm 0.6$ & (4) & 0.16 & $\mathrm{AB}$ \\
\hline Mark 618 & $0434-10$ & 5.4 & 0.036 & 14.5 & 2300 & (7) & $2.72 \pm 0.15$ & $(25)$ & 0.58 & - \\
\hline IRAS $04416+1215$ & $0441+12$ & 14.1 & 0.089 & 16.1 & 1670 & (16) & $2.96 \pm 0.50$ & (4) & 0.16 & $\mathrm{AB}$ \\
\hline IRAS $04576+0912$ & $0457+09$ & 13.5 & 0.037 & 16.6 & 1220 & (16) & & (4) & & $\mathrm{AB}$ \\
\hline IRAS 04596-2257 & $0459-22$ & 3.1 & 0.041 & 15.6 & 1500 & (11) & & & $* 0.51$ & - \\
\hline IRAS $05262+4432$ & $0526+44$ & 38.3 & 0.032 & 13.6 & 700 & (16) & & & 0.06 & $\mathrm{AB}$ \\
\hline RX J07527+2617 & $0749+26$ & 5.1 & 0.082 & 17.0 & 1000 & $(2)$ & $3.00 \pm 0.26$ & (2) & 0.16 & $\mathrm{AB}$ \\
\hline Mark 382 & $0752+39$ & 5.8 & 0.034 & 15.5 & 1500 & (18) & $3.09 \pm 0.23$ & $(33)$ & 0.45 & $\mathrm{AB}$ \\
\hline Mark 110 & $0921+52$ & 1.6 & 0.036 & 15.4 & 2120 & (6) & $2.35 \pm 0.05$ & $(29)$ & 1.69 & - \\
\hline Mark 705 & $0923+12$ & 4.0 & 0.028 & 14.9 & 1990 & (6) & $2.33 \pm 0.09$ & (33) & 1.25 & $\mathrm{AB}$ \\
\hline Mark 707 & $0934+01$ & 4.7 & 0.051 & 16.3 & 1320 & (6) & 2.40 & (29) & 0.46 & $\mathrm{AB}$ \\
\hline Mark 124 & $0945+50$ & 1.3 & 0.056 & 15.3 & 1400 & (18) & & & & $\mathrm{AB}$ \\
\hline Mark 1239 & $0949-01$ & 4.1 & 0.019 & 14.4 & 910 & $(20)$ & $2.94 \pm 0.14$ & $(25)$ & 0.05 & $\mathrm{AB}$ \\
\hline IRAS $09571+8435$ & $0957+84$ & 3.9 & 0.092 & 17.0 & 1120 & (16) & $1.39 \pm 0.40$ & $(4)$ & 0.07 & $\mathrm{AB}$ \\
\hline PG 1011-040 & $1011-04$ & 4.5 & 0.058 & 15.5 & 1440 & $(6)$ & & & & $\mathrm{AB}$ \\
\hline PG 1016+336 & $1016+33$ & 1.6 & 0.024 & 15.9 & 1600 & $(21)$ & & & & $\mathrm{AB}$ \\
\hline Mark 142 & $1022+51$ & 1.2 & 0.045 & 15.8 & 1620 & (6) & $3.15 \pm 0.11$ & $(14)$ & 1.75 & $\mathrm{AB}$ \\
\hline KUG 1031+398 & $1031+39$ & 1.4 & 0.042 & 15.6 & 1500 & $(24)$ & $4.15 \pm 0.10$ & (14) & 2.66 & $\mathrm{AB}$ \\
\hline RX J10407+3300 & $1037+33$ & 2.2 & 0.081 & 16.5 & 1700 & $(2)$ & $2.13 \pm 0.15$ & $(2)$ & 0.25 & $\mathrm{AB}$ \\
\hline Mark 734 & $1119+12$ & 2.7 & 0.049 & 14.6 & 1820 & (6) & $3.63 \pm 0.19$ & $(33)$ & 0.42 & $\mathrm{AB}$ \\
\hline Mark 739E & $1133+21$ & 2.2 & 0.030 & 14.1 & 900 & (17) & $2.43 \pm 0.14$ & $(33)$ & 0.49 & $\mathrm{AB}$ \\
\hline MCG 06.26.012 & $1136+34$ & 1.9 & 0.032 & 15.4 & 1685 & (13) & $2.77 \pm 0.08$ & (9) & 0.86 & $\mathrm{AB}$ \\
\hline Mark 42 & $1151+46$ & 1.9 & 0.024 & 15.4 & 670 & $(20)$ & $2.76 \pm 0.23$ & $(33)$ & 0.19 & $\mathrm{AB}$ \\
\hline NGC 4051 & $1200+44$ & 1.3 & 0.002 & 12.9 & 990 & $(22)$ & $2.84 \pm 0.04$ & (14) & 3.92 & $\mathrm{AB}$ \\
\hline PG $1211+143$ & $1211+14$ & 2.8 & 0.085 & 14.6 & 1860 & (6) & $3.03 \pm 0.15$ & (14) & 1.56 & $\mathrm{AB}$ \\
\hline Mark 766 & $1215+30$ & 1.8 & 0.012 & 13.6 & 2400 & $(20)$ & $2.79 \pm 0.11$ & $(14)$ & 4.71 & $\mathrm{AB}$ \\
\hline MS $12170+0700$ & $1216+07$ & 2.2 & 0.080 & 16.3 & & & & & & $\mathrm{AB}$ \\
\hline MS $12235+2522$ & $1223+25$ & 1.8 & 0.067 & 16.3 & 1730 & $(26)$ & $3.9 \pm 0.3$ & (5) & $* 0.52$ & $\mathrm{AB}$ \\
\hline IC 3599 & $1235+26$ & 1.4 & 0.021 & 15.6 & 1200 & (8) & $4.2 \pm 0.1$ & $(12)$ & 5.10 & $\mathrm{AB}$ \\
\hline PG $1244+026$ & $1244+02$ & 1.9 & 0.048 & 16.1 & 830 & (6) & $3.26 \pm 0.13$ & (14) & 1.30 & $\mathrm{AB}$ \\
\hline NGC 4748 & $1249-13$ & 3.6 & 0.014 & 14.0 & 1100 & (19) & $2.46 \pm 0.15$ & $(26)$ & 0.97 & $\mathrm{AB}$ \\
\hline Mark 783 & $1300+16$ & 2.0 & 0.067 & 15.6 & 1900 & $(20)$ & & & 0.29 & $\mathrm{AB}$ \\
\hline R 14.01 & $1338-14$ & 7.6 & 0.042 & 14.6 & 1790 & (15) & & & 0.31 & $\mathrm{AB}$ \\
\hline Mark 69 & $1343+29$ & 1.1 & 0.076 & 15.9 & 1500 & (18) & & & 0.26 & $\mathrm{AB}$ \\
\hline $2 \mathrm{E} 1346+2646$ & $1346+26$ & 1.1 & 0.059 & 16.5 & & & $2.68 \pm 0.2$ & $(23)$ & $*_{0.16}$ & $\mathrm{AB}$ \\
\hline PG $1404+226$ & $1404+22$ & 2.0 & 0.098 & 15.8 & 880 & (6) & $4.04 \pm 0.20$ & $(29)$ & 0.45 & $\mathrm{AB}$ \\
\hline Mark 684 & $1428+28$ & 1.5 & 0.046 & 14.7 & 1400 & $(21)$ & $2.4 \pm 0.2$ & $(12)$ & 0.58 & $\mathrm{AB}$ \\
\hline Mark 478 & $1440+35$ & 1.0 & 0.077 & 14.6 & 1450 & (6) & $3.06 \pm 0.03$ & (14) & 5.78 & $\mathrm{AB}$ \\
\hline PG $1448+273$ & $1448+27$ & 2.7 & 0.065 & 15.0 & 910 & (6) & $3.17 \pm 0.32$ & (28) & 0.78 & $\mathrm{AB}$ \\
\hline
\end{tabular}


Table 2. Continued.

\begin{tabular}{|c|c|c|c|c|c|c|c|c|c|c|}
\hline Name & Position & $N_{\mathrm{H}}$ & $z$ & $B$ & $F W$ & & $\Gamma$ & & $\mathrm{X}$ & \\
\hline IRAS $15091-2107$ & $1509-21$ & 8.8 & 0.044 & 14.8 & 1480 & (10) & & & 0.36 & - \\
\hline MS 15198-0633 & $1519-06$ & 12.4 & 0.084 & 14.9 & 1304 & (1) & $3.39 \pm 0.26$ & $(31)$ & 0.15 & $\mathrm{~A}$ \\
\hline Mark 486 & $1535+54$ & 1.8 & 0.038 & 14.8 & 1480 & (6) & & & $* 0.05$ & $\mathrm{AB}$ \\
\hline IRAS $15462-0450$ & $1546-04$ & 12.5 & 0.100 & 16.4 & & & & & & $\mathrm{AB}$ \\
\hline Mark 493 & $1557+35$ & 2.0 & 0.031 & 15.1 & 410 & $(20)$ & $2.84 \pm 0.14$ & $(33)$ & 0.52 & $\mathrm{AB}$ \\
\hline EXO 16524+3930 & $1652+39$ & 1.7 & 0.069 & 16.7 & 1000 & (3) & $2.7 \pm 0.2$ & (5) & 0.10 & $\mathrm{AB}$ \\
\hline B3 $1702+457$ & $1702+45$ & 2.2 & 0.060 & 15.1 & 490 & (16) & $2.37 \pm 0.18$ & (14) & 0.91 & $\mathrm{AB}$ \\
\hline RX J17450+4802 & $1743+48$ & 3.1 & 0.054 & 15.9 & 1600 & $(2)$ & $2.64 \pm 0.13$ & $(2)$ & 0.27 & B \\
\hline Kaz 163 & $1747+68$ & 4.4 & 0.063 & 15.0 & 1260 & (10) & $2.76 \pm 0.03$ & (14) & 0.21 & $\mathrm{AB}$ \\
\hline Mark 507 & $1748+68$ & 4.3 & 0.053 & 15.4 & 965 & (10) & $1.68 \pm 0.16$ & $(14)$ & $* 0.03$ & $\mathrm{AB}$ \\
\hline HS $1817+5342$ & $1817+53$ & 4.9 & 0.080 & 15.2 & & & & & 0.48 & $\mathrm{AB}$ \\
\hline HS $1831+5338$ & $1831+53$ & 4.9 & 0.039 & 15.9 & & & & & 0.06 & $\mathrm{AB}$ \\
\hline Mark 896 & $2043-02$ & 4.0 & 0.027 & 14.6 & 1330 & $(27)$ & $3.38 \pm 0.05$ & $(33)$ & 0.44 & $\mathrm{AB}$ \\
\hline MS $22102+1827$ & $2210+18$ & 6.2 & 0.079 & 16.7 & & & & & 0.13 & $\mathrm{AB}$ \\
\hline Akn 564 & $2240+29$ & 6.4 & 0.025 & 14.2 & 750 & $(27)$ & $3.47 \pm 0.07$ & $(14)$ & 3.84 & $\mathrm{AB}$ \\
\hline HS $2247+1044$ & $2247+10$ & 6.2 & 0.083 & 15.8 & & & & & 0.18 & $\mathrm{AB}$ \\
\hline Kaz 320 & $2257+24$ & 4.9 & 0.034 & 16.8 & 1800 & $(32)$ & & & 0.53 & $\mathrm{AB}$ \\
\hline
\end{tabular}

(Boroson \& Green 1992) and of $\mathrm{H} \beta$ and C III] $\lambda 1909$ (Wills et al. 2000) are strongly correlated; in two NLS1s, Leighly (2001) found that Si III] $\lambda 1892$ and C III] have the same width as the LILs; but, if Puchnarewicz et al. (1997) found that the $F W H M$ of $\mathrm{H} \beta$ and $\mathrm{Mg}$ II are also correlated, they concluded that that of C III] and Mg II are not. These observations support the idea of separate HIL and LIL emitting regions although it is not quite clear if $\mathrm{C}$ III] belongs to the HIL or to the LIL region.

The Fe II emission in most AGNs is too strong to be explained by photoionization (Phillips 1978b; Kwan et al. 1995); Fe II lines, and the other LILs as well, are more likely to be explained in the framework of collisional models where both the excitation of the lines and the ionization of the elements are due to collisions in a high density optically-thick medium illuminated mainly by hard X-rays; the Fe II emission region has a high density $\left(10^{10}<N_{\mathrm{e}}<10^{11}\right.$ or $\left.10^{12} \mathrm{~cm}^{-3}\right)$, a high column density $\left(N_{\mathrm{H}}>10^{24} \mathrm{~cm}^{-2}\right)$ and a low temperature (7500 < T < $10000 \mathrm{~K}$ ) (Collin-Souffrin et al. 1980; 1988b; Joly 1981; Clavel et al. 1983; Collin-Souffrin \& Lasota 1988; Kwan et al. 1995). There is a significant correlation between Si III]/C III] and $R_{4570}$; Si III and C III have similar ionization potentials, but Si III] has a critical density more than one order of magnitude larger than $\mathrm{C}$ III] $\left(1.1 \times 10^{11}\right.$ and $5 \times 10^{9} \mathrm{~cm}^{-3}$ respectively $)$; $\mathrm{Si} \mathrm{III]} / \mathrm{C} \mathrm{III]} \mathrm{is}$ thus a density indicator and becomes larger when density is higher; $\mathrm{Si} \mathrm{III]/C} \mathrm{III]} \mathrm{increases} \mathrm{with} \mathrm{increasing} N_{\mathrm{e}}$ up to $N_{\mathrm{e}}=10^{11} \mathrm{~cm}^{-3}$ (Aoki \& Yoshida 1999; Wills et al. 1999; Kuraszkiewicz et al. 2000). It seems therefore likely that the C III] line comes from the same high density region as $\mathrm{H} \beta$ and Fe II where it is at least partially suppressed by collisions.
HILs are emitted by low-pressure optically thin clouds (density of at most a few $10^{9} \mathrm{~cm}^{-3}$ ) illuminated by a rather soft continuum radiation (UV and soft X-rays) (Collin-Souffrin \& Lasota 1988). Collin-Souffrin et al. (1988a) suggested that these lines are produced in clouds undergoing predominantly outward motions along the system axis, the clouds receding from us being hidden by an opaque structure such as the accretion disk. The bipolar outflow could be a hydromagnetically driven wind accelerated radiatively and centrifugally away from the surface of the accretion disk (Emmering et al. 1992; Königl \& Kartje 1994; Bottorf et al. 1997; Murray \& Chiang 1998).

The broad Balmer lines exhibit a wide variety of profile shapes and a large range in width (Osterbrock \& Shuder 1982; de Robertis 1985; Crenshaw 1986; Stirpe 1991; Miller et al. 1992); they are often strongly asymmetric (Corbin 1995). The BLR consists of two components: one Intermediate Line Region (ILR) with line width $\sim 2000 \mathrm{~km} \mathrm{~s}^{-1} F W H M$, with the peak within a few hundred kilometers per second of the systemic redshift, and a Very Broad Line Region (VBLR) with lines of width $>7000 \mathrm{~km} \mathrm{~s}^{-1}$ and blueshifted by more than $1000 \mathrm{~km} \mathrm{~s}^{-1}$; differences in the relative strengths of these components account for much of the diversity of broad line profiles (Wills et al. 1993; Brotherton et al. 1994; Corbin 1995, 1997; Francis et al. 1992). The spectra of the VBLR and ILR are very different (Brotherton et al. 1994); the VBLR and the ILR can probably be identified with the HIL and LIL regions respectively (Puchnarewicz et al. 1997). This is confirmed by variability studies: the profile of the broad emission lines are variable; many of them can be described by two Gaussian components that are nearly stationary in wavelength, and which vary independently of one another in relative flux (Peterson et al. 1999). This is the 

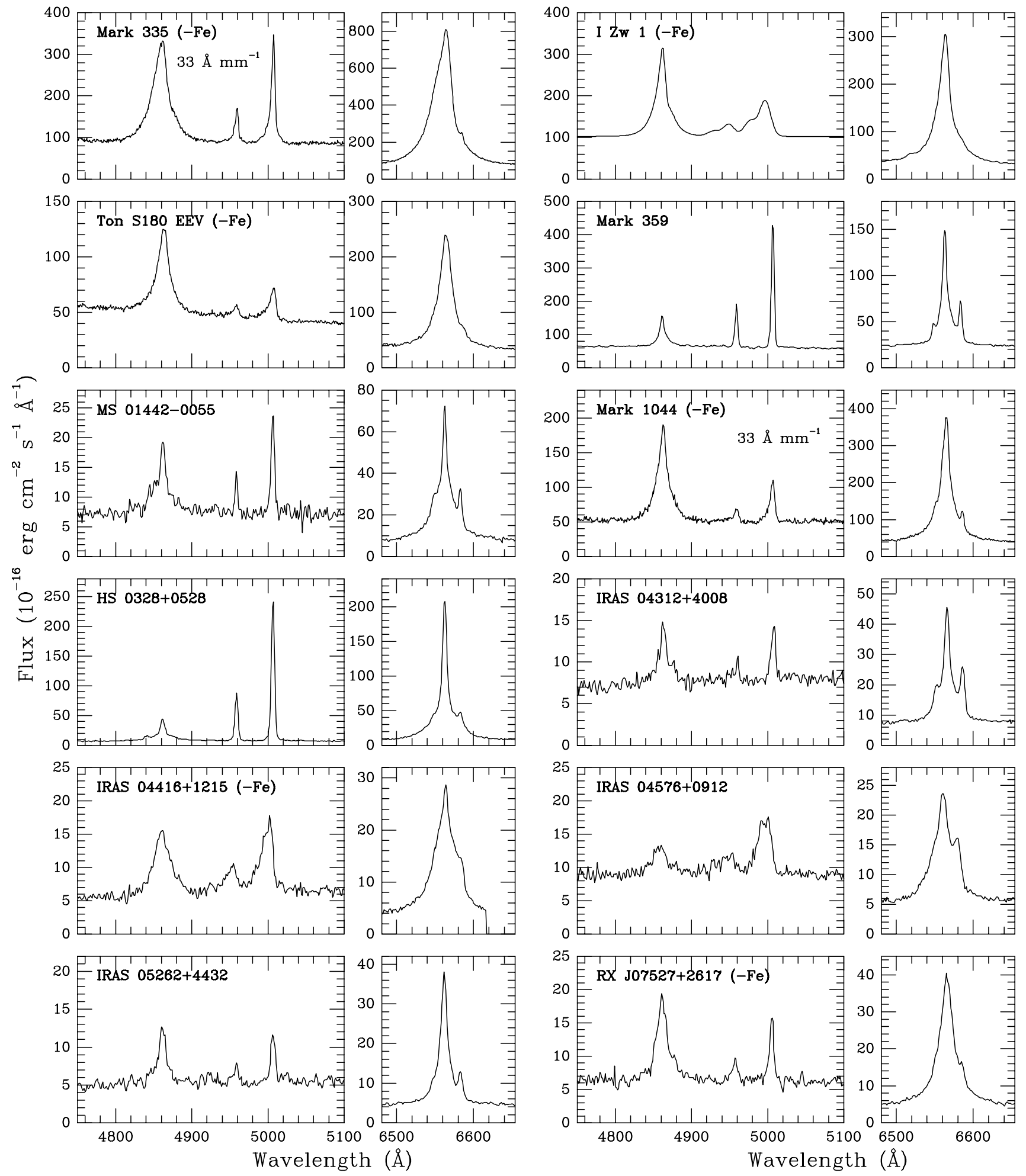

Fig. 1. Deredshifted blue and red spectra of the observed NLS1s.

case for NGC 5548 (Dumont et al. 1998) and NGC 3516 (Goad et al. 1999) in which the emission lines are best explained by the superposition of an emission line cloud with variable lines and another which shows no variability; the emission spectrum of the non variable cloud is dominated by Balmer lines and Fe II emission. In the case of PG 1416-129, the broad $\mathrm{H} \beta$ component $\left(4000 \mathrm{~km} \mathrm{~s}^{-1}\right.$
$F W H M)$ is strongly variable while the very broad component (13000 $\left.\mathrm{km} \mathrm{s}^{-1} F W H M\right)$ has a much smaller amplitude (Sulentic et al. 2000b).

LILs could be produced in the outer part of the disk itself as a result of energy reflected from the flow above the disk (Collin-Souffrin et al. 1988a). The profile of the lines produced in a disk are generally not double peaked, except 

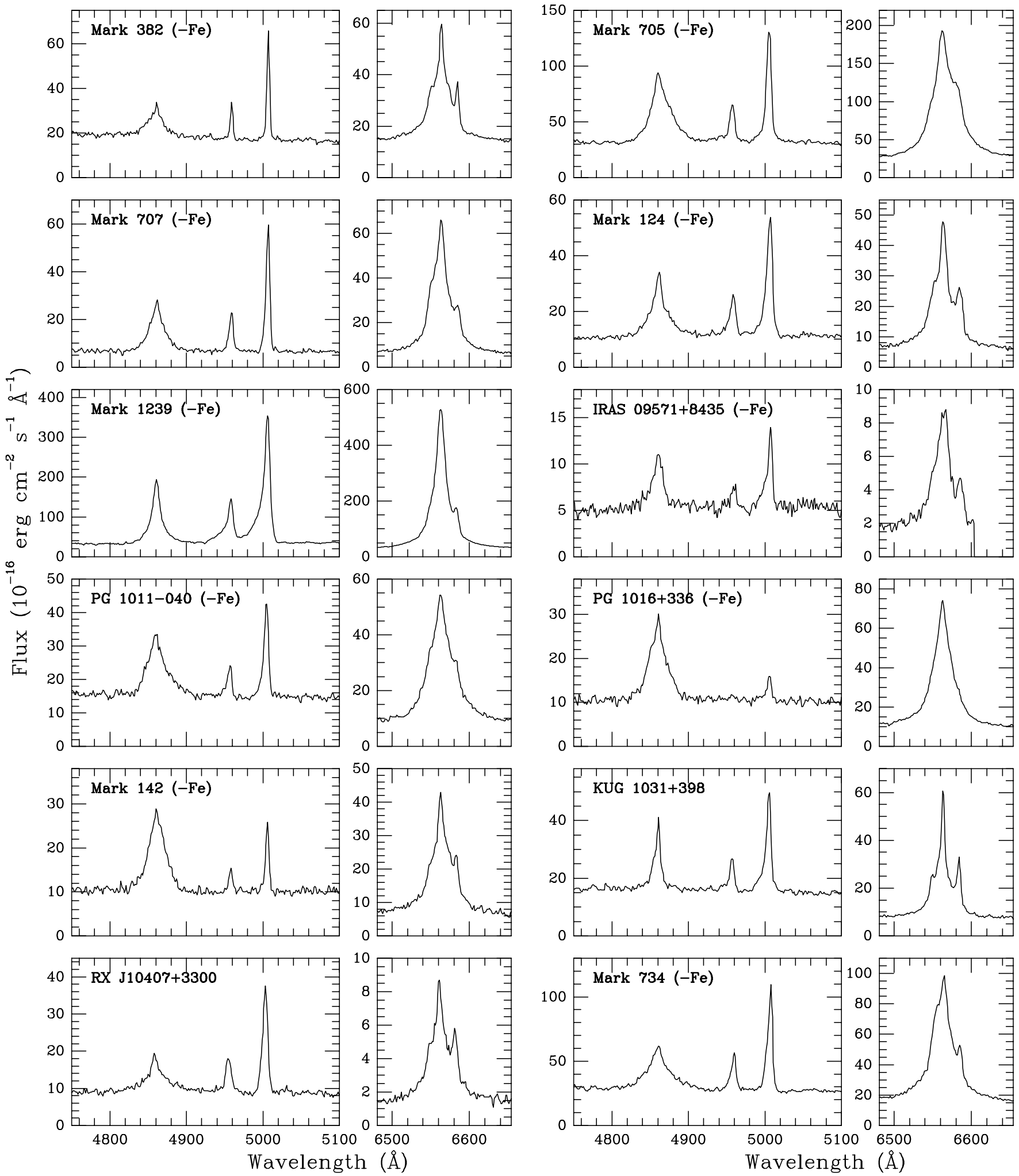

Fig. 2. Deredshifted blue and red spectra of the observed NLS1s (continued).

if the radius of the disk is small $\left(\sim 10^{3} R_{\mathrm{G}}\right.$ where $R_{\mathrm{G}}$ is the gravitational radius of the central $\mathrm{BH}$ ), in which case the line intensities are small, the line profiles are U-shaped and very broad; if the disk radius is large $\left(>10^{4} R_{\mathrm{G}}\right)$, the line intensities are large and their profile generally single peaked; the spectrum consists mainly of LILs and can be a major part of the broad line emission (Dumont \& CollinSouffrin 1990); these lines are, under certain conditions, more similar to a Lorentzian than to a Gaussian (see their Fig. 4b). 

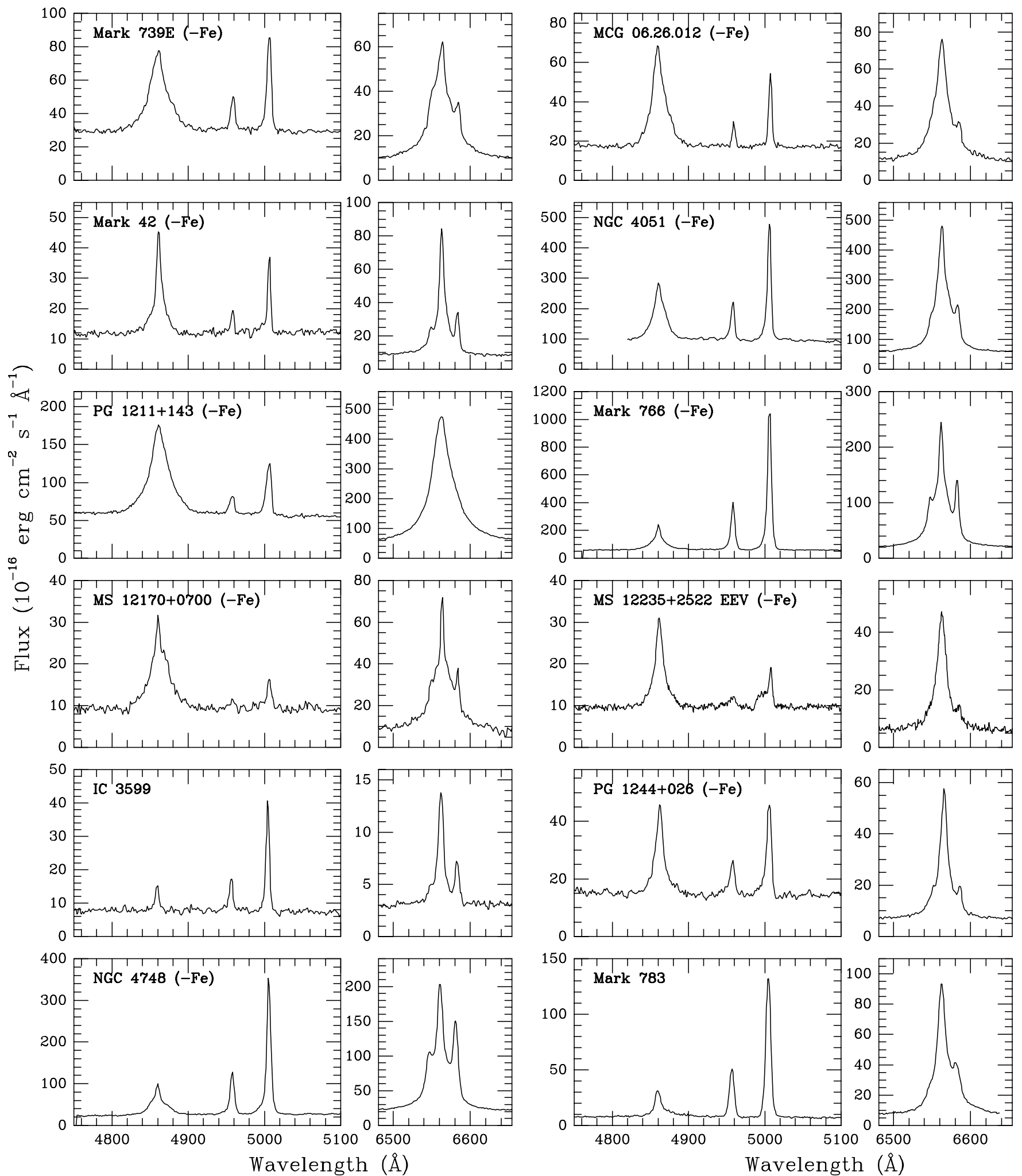

Fig. 3. Deredshifted blue and red spectra of the observed NLS1s (continued).

\subsubsection{On the profile of the NLS1 broad lines}

According to Moran et al. (1996) and Leighly (1999b), many NLS1s have symmetric emission lines with more nearly Lorentzian than Gaussian profiles. However
Rodríguez-Ardila et al. (2000b) claimed that Lorentzian profiles are not suited to represent the NLS1 broad emission-lines; this conclusion is based on the fact that these authors were unable to get a good fit when using a single Lorentzian for each of the emission lines; however, 

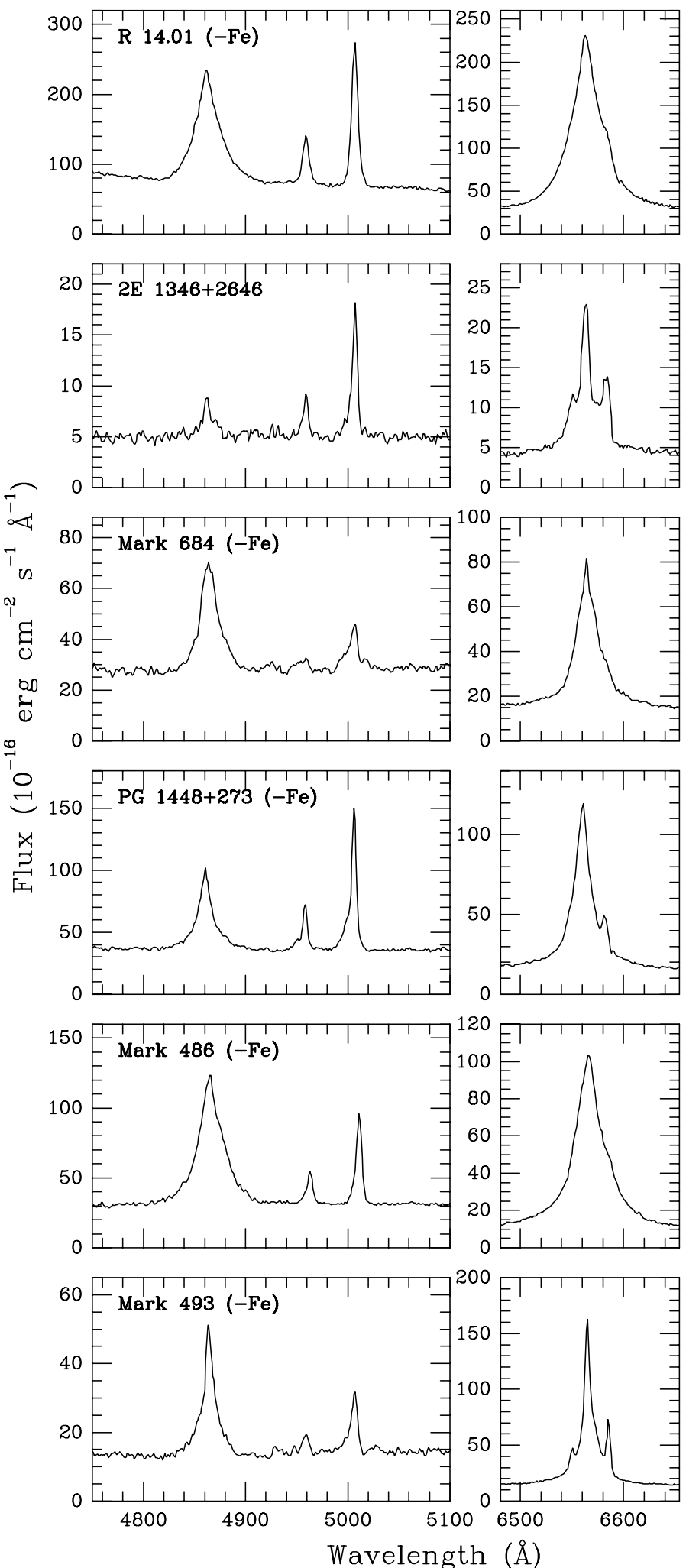
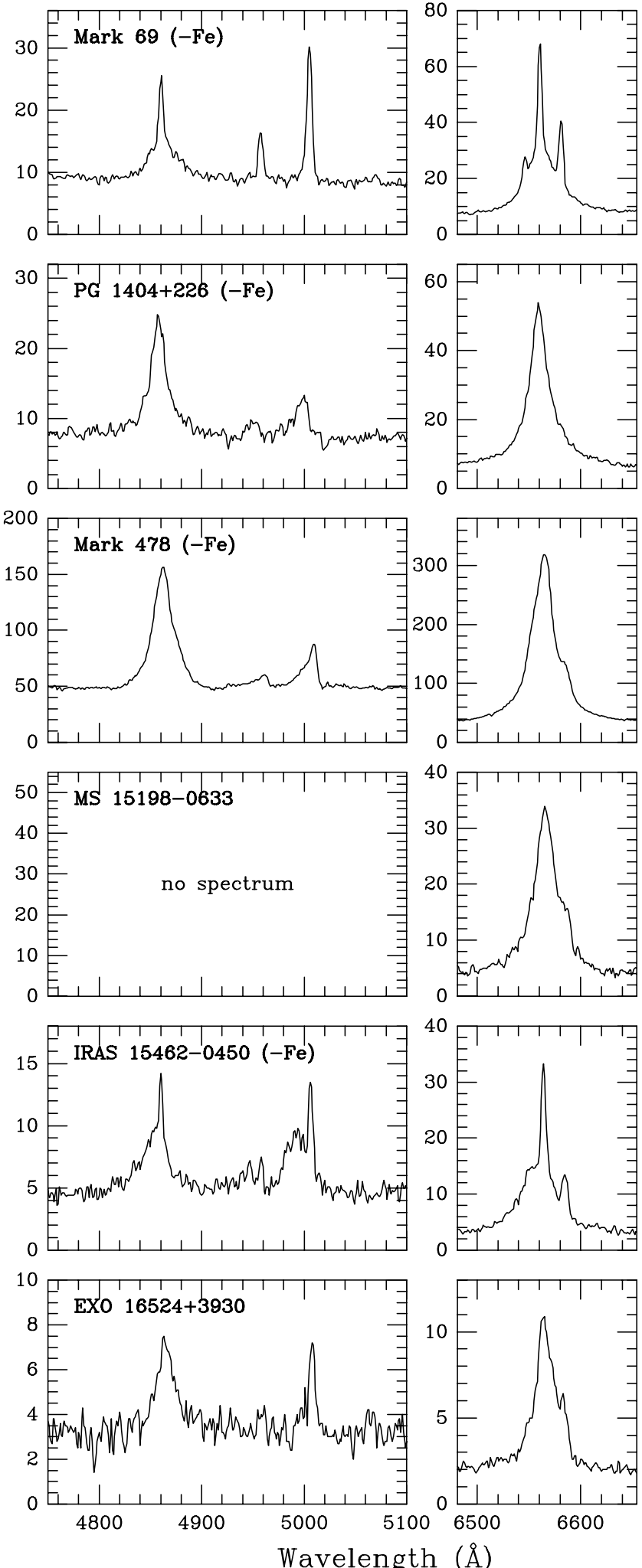

Fig. 4. Deredshifted blue and red spectra of the observed NLS1s (continued).

there is a major inconsistency in their procedure: they assumed that the Balmer lines (either $\mathrm{H} \alpha$ or $\mathrm{H} \beta$ ) had a pure Lorentzian profile, not allowing for the presence of a narrow component. It so happens that one of the objects they present as an example of the poor results obtained when fitting the emission lines with Lorentzians (Mark 1239) is one of those for which we obtain a very good fit using a Lorentzian profile for the broad Balmer line components in addition to a narrow component having the same Gaussian profile as the forbidden lines. 

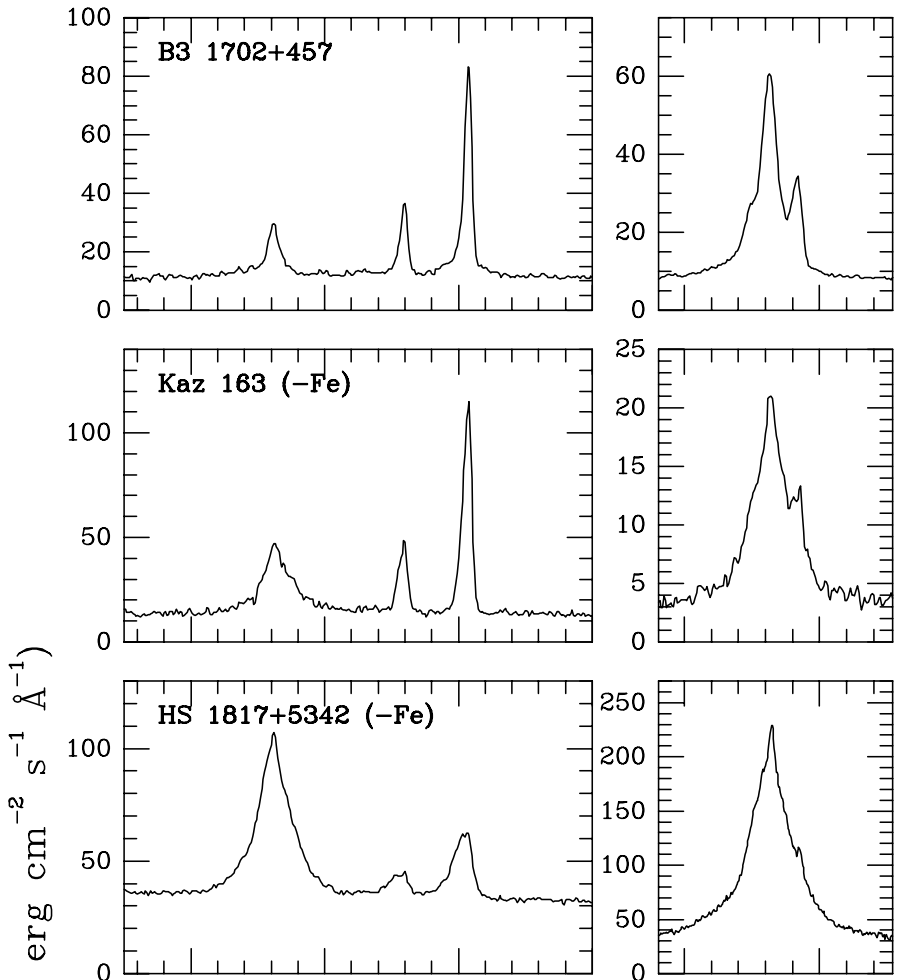

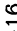
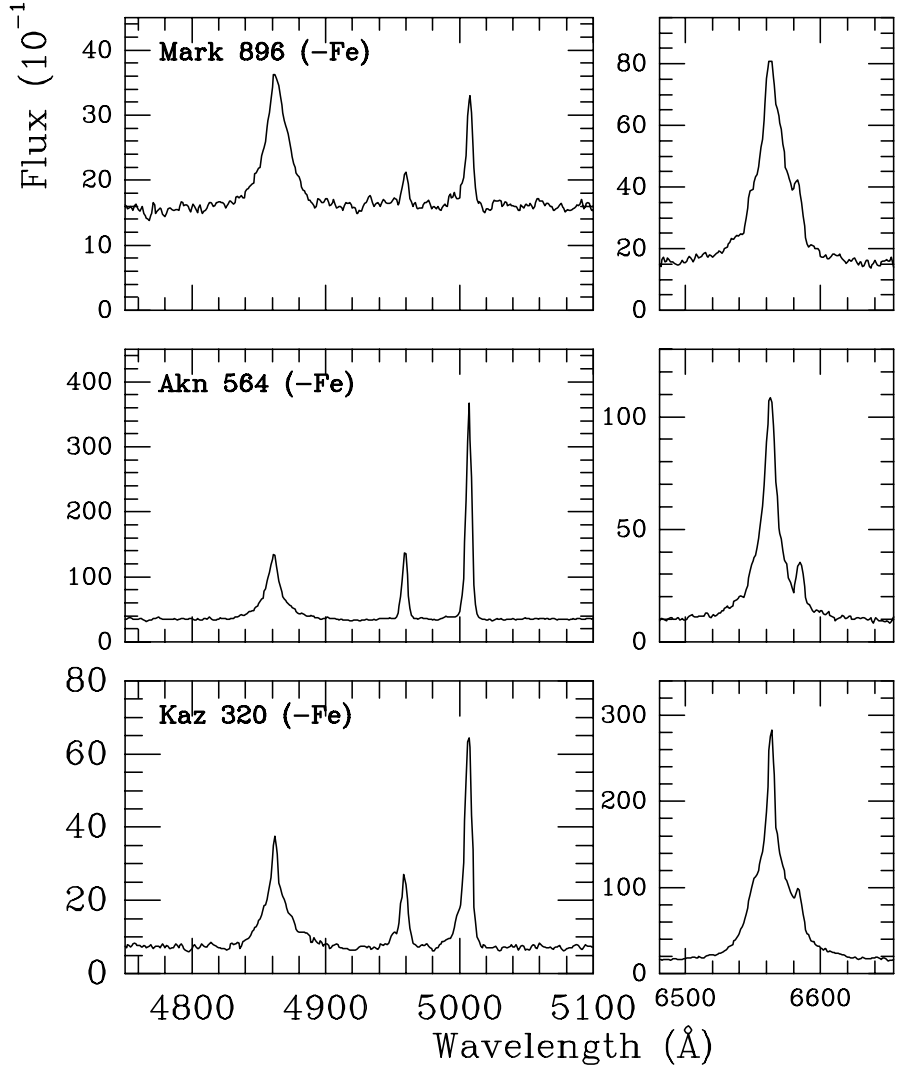
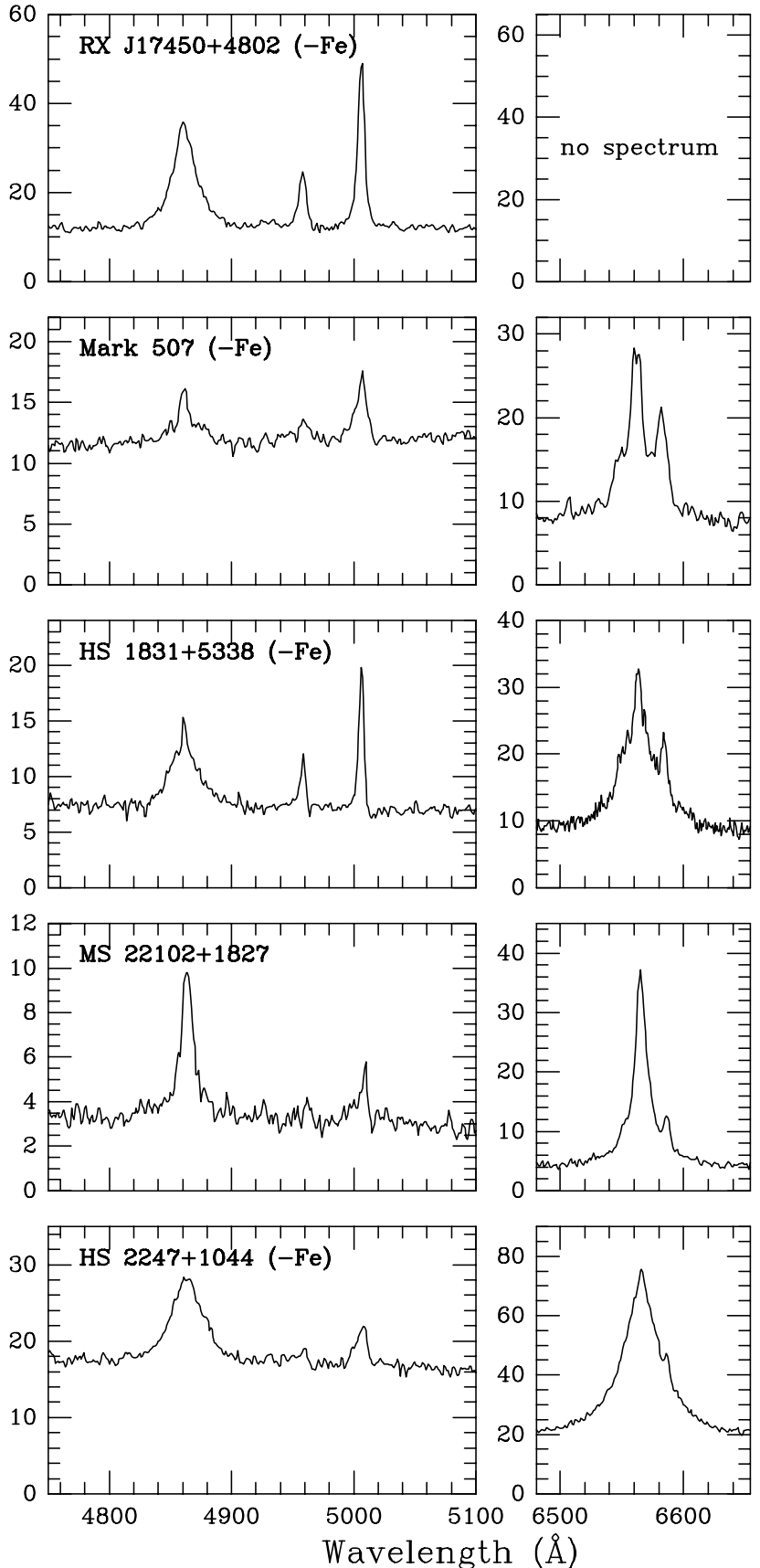

Fig. 5. Deredshifted blue and red spectra of the observed NLS1s (continued).

Although not all broad Balmer components in our sample are well fitted by a single Lorentzian, this is true in most cases (Table 3 lists, for all observed objects, the $F W H M$ of the $\mathrm{H} \alpha$ and $\mathrm{H} \beta$ broad components obtained by using a single Lorentzian). As an illustration, we show in Fig. 7 the blue and red spectra of four objects fitted by a single broad Gaussian and by a single broad Lorentzian; the narrow lines have been fitted either by a single Gaussian or by two Gaussians. In three cases, the fit of the red spectra is significantly better with a 

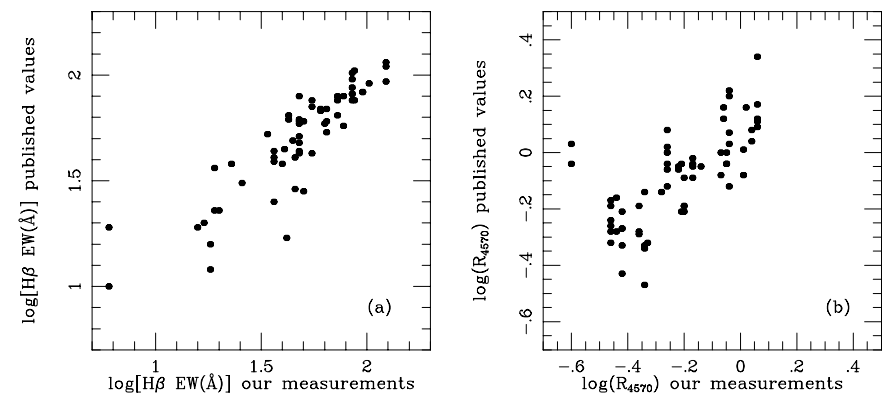

Fig. 6. a) Comparison between our measurements of the $\mathrm{H} \beta$ EW and the published ones. b) Comparison between our measurements of $R_{4570}$ and the published ones.

Lorentzian than with a Gaussian, as judged from the residuals. The improvement of the fit of the blue spectra with a Lorentzian is significant only for PG $1244+026$. But, in all cases, the Lorentzian fit leads to $\lambda 6583 / \mathrm{H} \alpha$ and $\lambda 5007 / \mathrm{H} \beta$ ratios which are more similar to the values expected for Seyfert 2 nebulosities (see below).

The use of a Lorentzian rather than a Gaussian leads, for the NLR of most NLS1s, to line ratios typical of classical Seyfert 2s which strongly confirms that these broad Balmer components have a profile which is genuinely more similar to a Lorentzian. We give in Table 4 the FWHM of the broad Gaussian and Lorentzian profiles for these four objects; we first note that the FWHM of the $\mathrm{H} \alpha$ and $\mathrm{H} \beta$ Lorentzians are equal within $\sim 10 \%$, while the $\mathrm{H} \alpha$ $F W H M$ of the Gaussians are on average $50 \%$ larger than the $\mathrm{H} \beta \quad F W H M$; this probably reflects the better quality of the fits obtained with Lorentzians. The FWHM of the Gaussians are systematically larger than the FWHM of the Lorentzians; in particular, HS 2247+1044 would not be classified as an NLS1 from a Gaussian fit.

The fact that the broad Balmer lines in NLS1s have a nearly Lorentzian profile seems to suggest that they are produced in a large disk without the contribution from an outflowing component. However, the high ionization UV emission lines in the spectrum of NLS1s appear to be significantly broader than $\mathrm{H} \beta$ (Rodríguez-Pascual et al. 1997; Kuraszkiewicz et al. 2000; Wills et al. 2000). In addition, Leighly (2001) showed that the high-ionization UV emission lines in two NLS1s (IRAS 13224-3809 and 1H 0707-495) are much broader and strongly blueshifted than the low-ionization lines. These observations suggest that, in NLS1s, the outflowing component exists but is relatively weak, its contribution being negligible in the optical range.

\subsection{The narrow line region}

\subsubsection{The line ratios of the narrow line region}

As we have seen above, for all objects for which a single Lorentzian gave a good fit to the broad Balmer component, we also made a fit with a single Gaussian. In this way, we obtained two sets of line ratios $(\lambda 5007 / \mathrm{H} \beta$ and $\lambda 6583 / \mathrm{H} \alpha$ ) which are plotted in Fig. 8 (when the signal to noise ratio is low, the fit with a single broad $\mathrm{H} \beta$ Lorentzian sometimes gives a low value for the $\lambda 5007 / \mathrm{H} \beta$ ratio; in these cases, when forcing this ratio to be equal to 10 , the fit is not worse as illustrated for IRAS $05262+4432$ in Fig. 9). The left panel shows the ratios obtained by fitting the broad Balmer component with a Gaussian; the right panel, the ratios obtained with a Lorentzian. While, in the left panel, many points are located in the H II region or composite domain (Gonçalves et al. 1999a), in the right panel, most of the points are in the Seyfert 2 region; one object (HS 0328+0528) belongs to the rare class of weak-[N II] Seyfert galaxies (Gonçalves et al. 1999a) while six (Mark 42, MS 12170+0700, Mark 69, Mark 684, IRAS 15462-0450 and Mark 507) have an emission line component which falls in or near the H II region domain and is a genuine H II region; they are shown as open circles; these objects are most probably composite type objects similar to those described by Véron et al. (1981b) and Gonçalves et al. (1999a). The fit of the broad component of I Zw 1 with a single component is clearly unsatisfactory; we got a better fit with two Lorentzians which puts the representative point of the narrow line region in the Seyfert 2 area.

In contrast, Rodríguez-Ardila et al. (2000b) found that the $\lambda 5007 / \mathrm{H} \beta_{\mathrm{n}}$ ratio emitted in the NLR of NLS1s varies from 1 to 5 , instead of $\sim 10$ for BLS1s. This is most probably due to the fact that they modelled the broad Balmer component with a Gaussian rather than a Lorentzian. On the other hand, Nagao et al. (2001) showed that the line ratios $[\mathrm{O}$ I $] \lambda 6300 /[\mathrm{O}$ III $] \lambda 5007$ and $[\mathrm{O}$ III $] \lambda 4363 /[\mathrm{O}$ III $] \lambda 5007$ are statistically indistinguishable in NLS1s and BLS1s.

\subsubsection{The kinematics of the narrow line region}

The majority of the profiles of the [O III] lines in Seyfert 1s are markedly asymmetric, exhibiting a sharper falloff to the red than to the blue (Heckman et al. 1981; Vrtilek \& Carleton 1985; Whittle 1985a; Veilleux 1991b); these profiles often have a two component structure with a narrow core superposed on a broader, blueshifted base (Heckman et al. 1981; Leighly 1999b); it seems that the velocity of the core is equal to the systemic velocity of the galaxy (Heckman et al. 1981, 1984; Whittle 1985a).

Many Seyferts have an "ionization cone" appearing as either one- or two-sided structures emanating from the active nucleus; when single (one-sided) cones are seen, they generally project against the far side of the galaxy disk, suggesting that the counter cone is present but obscured by dust in the disk (Pogge 1989; Wilson et al. 1993; Wilson 1994). Colbert et al. (1996) suggested that more than $25 \%$ of Seyfert galaxies have good evidence (from the kinematics of the $\mathrm{H} \alpha$ and [N II] lines) for minor axis galactic outflows. Crenshaw et al. (1999) showed, from the observation of UV absorption lines (mainly C IV and N V), that large scale outflows with a velocity of a few hundreds $\mathrm{km} \mathrm{s}^{-1}$ are common in Seyfert galaxies. High spatial resolution 

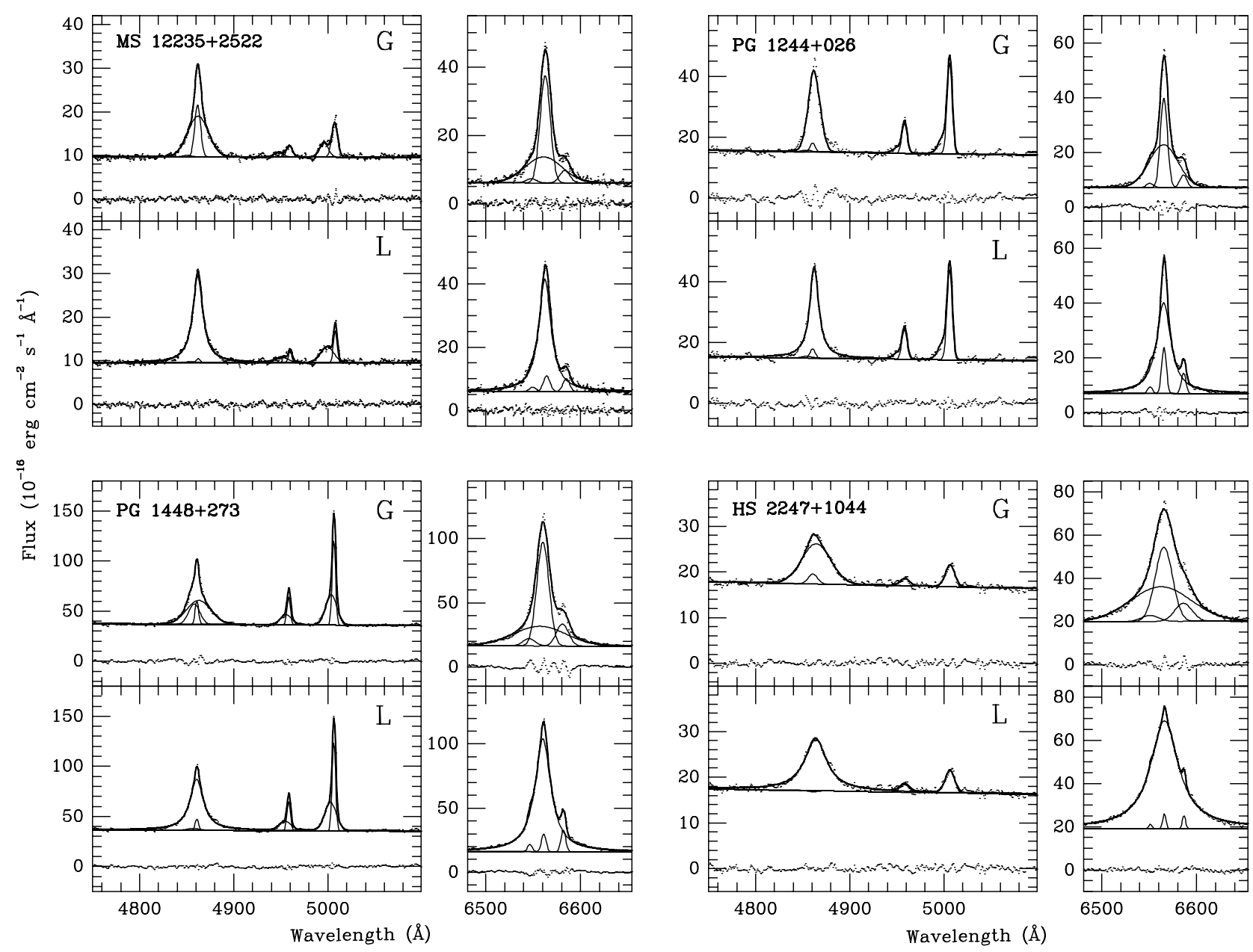

Fig. 7. Deredshifted blue and red spectra of four NLS1 galaxies in our sample. The data points were fitted by a set of narrow Gaussian profiles, reproducing the narrow emission lines, plus a Gaussian (G) or Lorentzian (L) profile, to fit the broad Balmer component. The individual profiles are represented by a thin solid line, the total fit by a thick solid line and the differences between the data and the fit (the residuals) by the lower dotted line.

spectroscopic observations of the nuclear emission lines of NGC 1068 (Crenshaw \& Kraemer 2000) and NGC 4151 (Crenshaw et al. 2000; Kaiser et al. 2000) give strong support for biconical radial outflows in these objects.

Whittle et al. (1988), Storchi-Bergmann et al. (1992), Arribas \& Mediavilla (1993) and Kaiser et al. (2000) showed that the AGN NLRs have two line-emitting constituents: the "[O III] components" (the broad blueshifted bases of the [O III] lines; Heckman et al. 1981; Vrtilek 1985; Whittle 1992; Christopoulou et al. 1997), to be identified with the biconical structure and which are outflowing from the nucleus with a speed of a few hundreds $\mathrm{km} \mathrm{s}^{-1}$, and the "ambient [O III] emission" which is more widely distributed and shows normal galactic rotation. A detailed kinematic study of the emission line gas in the Seyfert galaxy NGC 2992 shows the presence of both a disk component which is well modeled by pure circular rotation and an outflowing component distributed into two wide cones (Veilleux et al. 2001). Smith (1993) suggested that the "[O III] component" could be accelerated outwards by a supersonic wind generated by the active nucleus. The relative intensity of the two components varies widely from object to object.

High-ionization lines ([Fe VII], $[\mathrm{Fe} \mathrm{X}]$ and $[\mathrm{Fe} \mathrm{XI}]$ ) are often present in emission in NLS1s; these coronal lines tend to be blueshifted relative to, and broader than, the low-ionization lines; the systematic blueshift indicates an outflow of the gas emitting these features (Grandi 1978; Erkens et al. 1997).

There is a strong correlation between the [O III $] \lambda 5007$ line width and luminosity (Whittle 1985b). The [O III] FWHM is correlated with the absolute magnitude of the galaxy bulge (Véron \& Véron-Cetty 1986) and the observed galaxy rotation (Véron 1981; Whittle 1992) showing that gravity plays a dominant role in the NLR of most objects, at least in the "ambient [O III] emission" region which dominates in most AGNs.

For a number of objects in our sample (30), the fit of the [O III] lines with a single Gaussian was poor, either because of the simultaneous presence on the slit of 
Table 3. Line properties of the 59 observed NLS1s (H II components have been systematically removed). Column 1: name, Col. 2: $R_{4570}$, ratio of the Fe II to the total $\mathrm{H} \beta$ fluxes, Cols. 3 and $4: F W H M$ (in $\mathrm{km} \mathrm{s}^{-1}$ ) of the broad $\mathrm{H} \alpha$ and $\mathrm{H} \beta$ lines fitted with a Lorentzian profile, Col. 5: $E W(\AA)$ of the broad $\mathrm{H} \beta$ component, Col. 6: $R_{5007}$, ratio of the $\lambda 5007$ to the total $\mathrm{H} \beta$ fluxes, Col. 7: FWHM (corrected for instrumental broadening) of the [O III] line, Cols. 8 and 9: $F W H M$ of the individual components (F1 and F2) of the [O III] lines, Col. 10: velocity difference between the two [O III] components (broad-narrow), Col. 11: intensity ratio of the two [O III] components (broad/narrow). a: a good fit can only be obtained with two broad components, a Lorentzian and a Gaussian or two Lorentzians. b: the broad $\mathrm{H} \beta$ component is weak.

\begin{tabular}{|c|c|c|c|c|c|c|c|c|c|c|}
\hline \multirow[t]{2}{*}{ Name } & \multirow[t]{2}{*}{$R_{4570}$} & \multicolumn{2}{|c|}{$F W H M(L)$} & \multirow{2}{*}{$\begin{array}{c}E W \\
\mathrm{H} \beta_{\mathrm{br}}\end{array}$} & \multirow[t]{2}{*}{$R_{5007}$} & \multirow{2}{*}{$\begin{array}{r}F W H M \\
\lambda 5007\end{array}$} & \multirow[t]{2}{*}{$F W 1$} & \multirow[t]{2}{*}{$F W 2$} & \multirow[t]{2}{*}{$\Delta V$} & \multirow[t]{2}{*}{$F 2 / F 1$} \\
\hline & & $\mathrm{H} \alpha$ & $\mathrm{H} \beta$ & & & & & & & \\
\hline Mark 335 & 0.38 & $\mathrm{a}$ & $\mathrm{a}$ & 86 & 0.25 & 280 & 245 & 910 & -190 & 1.23 \\
\hline $\mathrm{IZw} 1$ & 1.14 & $\mathrm{a}$ & $\mathrm{a}$ & 48 & 0.44 & 1660 & 1040 & 1070 & -1180 & 0.43 \\
\hline Ton S180 & 1.03 & 1250 & 1085 & 46 & 0.18 & 675 & 435 & 1060 & -465 & 1.00 \\
\hline Mark 359 & $<0.5$ & 830 & 900 & 18 & 1.32 & 180 & & & & \\
\hline MS 01442-0055 & $<0.6$ & 1260 & 1100 & 35 & 0.35 & 240 & & & & \\
\hline Mark 1044 & 0.61 & 905 & 1010 & 63 & 0.15 & 420 & 335 & 720 & -340 & 0.69 \\
\hline HS $0328+0528$ & $<0.6$ & 1455 & 1590 & 79 & 1.67 & 220 & & & & \\
\hline IRAS $04312+4008$ & $<1.4$ & 1060 & 860 & 16 & 0.35 & 380 & & & & \\
\hline IRAS $04416+1215$ & 1.14 & 1500 & 1470 & 55 & 0.67 & 1320 & 650 & 1790 & -480 & 1.95 \\
\hline IRAS $04576+0912$ & $<1.0$ & 1100 & 1210 & 13 & 1.40 & 1290 & 380 & 1260 & -500 & 6.40 \\
\hline IRAS $05262+4432$ & 1.09 & 695 & 740 & 23 & 0.35 & 365 & & & & \\
\hline RX J07527+2617 & 0.71 & 1080 & 1185 & 50 & 0.21 & 400 & & & & \\
\hline Mark 382 & $<0.8$ & 1270 & 1280 & 20 & 0.56 & 155 & & & & \\
\hline Mark 705 & 0.36 & 1745 & 1790 & 85 & 0.41 & 365 & 365 & 1630 & -290 & 0.62 \\
\hline Mark 707 & 0.47 & 1180 & 1295 & 102 & 0.53 & 315 & & & & \\
\hline Mark 124 & 0.60 & 1645 & 1840 & 43 & 0.72 & 540 & 380 & 935 & -335 & 0.75 \\
\hline Mark 1239 & 0.63 & 905 & 1075 & 78 & 1.29 & 630 & 400 & 1395 & -475 & 1.16 \\
\hline IRAS $09571+8435$ & 1.05 & 1270 & 1185 & 26 & 0.53 & 430 & 240 & 845 & -370 & 1.07 \\
\hline PG 1011-040 & 0.46 & 1370 & 1455 & 41 & 0.33 & 400 & & & & \\
\hline PG 1016+336 & 0.60 & 1205 & 1590 & 65 & 0.06 & 315 & & & & \\
\hline Mark 142 & 0.92 & 1335 & 1370 & 60 & 0.14 & 260 & & & & \\
\hline KUG $1031+398$ & $<1.5$ & 1225 & 935 & 17 & 0.93 & 1000 & 315 & 1115 & -280 & 1.27 \\
\hline RX J10407+3300 & $<0.6$ & 1425 & 1985 & 32 & 0.75 & 460 & & & & \\
\hline Mark 734 & 0.67 & 1345 & 1825 & 45 & 0.49 & 450 & 180 & 525 & -230 & 1.41 \\
\hline Mark 739E & 0.61 & 1415 & 1615 & 63 & 0.23 & 380 & & & & \\
\hline MCG 06.26.012 & 0.52 & 1070 & 1145 & 83 & 0.14 & 220 & & & & \\
\hline Mark 42 & 0.90 & 805 & 865 & 36 & & 220 & & & & \\
\hline NGC 4051 & 0.25 & 970 & 1120 & 50 & 0.55 & 300 & 200 & 665 & -175 & 0.65 \\
\hline
\end{tabular}

a H II region (6), or because the lines are asymmetrical with a blue wing (24); in these last cases, we fitted them with two Gaussians following the procedure described in Sect. 2; as a result, we found a red component with a $F W H M$ in the range $180-650 \mathrm{~km} \mathrm{~s}^{-1}$ and a blue component with a $F W H M$ in the range $525-1790 \mathrm{~km} \mathrm{~s}^{-1}$, blueshifted by 90 to $570 \mathrm{~km} \mathrm{~s}^{-1}$ with respect to the red component (Table 3 ; in this table, the [O III] FWHM have been corrected for the instrumental profile); there is one exception, I Zw 1, for which the red component is exceptionally broad (1040 $\left.\mathrm{km} \mathrm{s}^{-1} F W H M\right)$ and the blue component is blueshifted by $1180 \mathrm{~km} \mathrm{~s}^{-1}$. This is in agreement with previous findings.

For the objects requiring an additional [O III] component, the associated $\mathrm{H} \beta$ line was often very weak; as a result, the fitting routine sometimes gave a negative flux; in such cases, we set the ratio $\lambda 5007 / \mathrm{H} \beta$ to 10 , the mean value for Seyfert galaxies. This is a common procedure used to prevent the fitting routine from yielding non-physical values whenever the line intensities are too small to be disentangled from the noise.
The soft X-ray photon index is available for 41 of the objects for which we have a blue spectrum. Twenty of them have $\Gamma>2.9,21$ have a smaller value; among the 20 objects with a steep soft X-ray spectrum, 12 have a blueshifted [O III] component, while only eight of the other set have such a component; moreover, two of the eight steep X-ray spectrum objects without a blueshifted component (PG 1404+226 and RXS J07527+2617) have an optical spectrum with a poor signal to noise ratio and relatively broad [O III] lines (950 and $400 \mathrm{~km} \mathrm{~s}^{-1}$ respectively) so that the presence of a blueshifted component could have been overlooked. So there is a weak, statistically unsignificant, trend for the objects with a soft X-ray excess to have an outflowing [O III] component. It is interesting to note that Erkens et al. (1997) have found that strong coronal lines occur predominantly in objects with the steepest soft X-ray spectra and that these lines are relatively broad and blueshifted. The FWHM and velocity shifts are comparable for the blueshifted [O III] lines and the coronal lines. Could these lines all come from the same emitting clouds? 
Table 3. Continued.

\begin{tabular}{|c|c|c|c|c|c|c|c|c|c|c|}
\hline \multirow[t]{2}{*}{ Name } & \multirow[t]{2}{*}{$R_{4570}$} & \multicolumn{2}{|c|}{$F W H M(L)$} & \multirow{2}{*}{$\begin{array}{c}E W \\
\mathrm{H} \beta_{\mathrm{br}}\end{array}$} & \multirow[t]{2}{*}{$R_{5007}$} & \multirow{2}{*}{$\begin{array}{r}F W H M \\
\lambda 5007\end{array}$} & \multirow[t]{2}{*}{$F W 1$} & \multirow[t]{2}{*}{$F W 2$} & \multirow[t]{2}{*}{$\Delta V$} & \multirow[t]{2}{*}{$F 2 / F 1$} \\
\hline & & $\mathrm{H} \alpha$ & $\mathrm{H} \beta$ & & & & & & & \\
\hline PG $1211+143$ & 0.44 & 1400 & 1975 & 95 & 0.12 & 510 & & & & \\
\hline Mark 766 & 0.35 & 1150 & 1630 & 55 & 1.83 & 330 & 220 & 710 & -90 & 0.73 \\
\hline MS $12170+0700$ & 0.65 & 1405 & 1765 & 79 & & 365 & & & & \\
\hline MS $12235+2522$ & 0.62 & 705 & 800 & 43 & 0.24 & 875 & 240 & 905 & -570 & 1.34 \\
\hline IC 3599 & & 500 & - & 6 & 3.23 & 280 & & & & \\
\hline PG $1244+026$ & 1.09 & 820 & 740 & 36 & 0.47 & 415 & 330 & 740 & -390 & 0.40 \\
\hline NGC 4748 & 0.55 & 1400 & 1565 & 65 & 1.34 & 365 & 295 & 1170 & -150 & 0.49 \\
\hline Mark 783 & $<0.5$ & 1510 & 1655 & 42 & 2.29 & 430 & & & & \\
\hline R 14.01 & 0.44 & 1470 & 1605 & 85 & 0.28 & 430 & & & & \\
\hline Mark 69 & 0.59 & 1445 & 1925 & 34 & & 315 & & & & \\
\hline $2 \mathrm{E} 1346+2646$ & & 1235 & $\mathrm{~b}$ & & 2.40 & 330 & 180 & 950 & -105 & 1.07 \\
\hline PG $1404+226$ & 0.85 & 1015 & 1120 & 65 & 0.19 & 950 & & & & \\
\hline Mark 684 & 0.91 & $\mathrm{a}$ & 1150 & 40 & 0.14 & 1290 & & & & \\
\hline Mark 478 & 0.55 & 1190 & 1270 & 72 & 0.17 & 920 & 365 & 1230 & -475 & 2.05 \\
\hline PG $1448+273$ & 0.73 & 915 & 1050 & 36 & 0.61 & 315 & 155 & 890 & -215 & 1.11 \\
\hline MS 15198-0633 & & 1115 & - & - & & & & & & \\
\hline Mark 486 & 0.46 & 1400 & 1680 & 123 & 0.13 & 400 & & & & \\
\hline IRAS $15462-0450$ & 0.59 & 1830 & 1615 & 41 & 0.62 & 1600 & & & & \\
\hline Mark 493 & 0.87 & 870 & 740 & 46 & 0.26 & 450 & 315 & 845 & -400 & 0.75 \\
\hline EXO 16524+3930 & $<0.9$ & 1025 & 1355 & 39 & 0.21 & 400 & & & & \\
\hline B3 $1702+457$ & $<1.2$ & 930 & 975 & 19 & 2.03 & 365 & 295 & 1200 & -280 & 0.56 \\
\hline RX J17450+4802 & 0.78 & - & 1355 & 48 & 0.45 & 400 & & & & \\
\hline Kaz 163 & 0.35 & 1325 & 1875 & 87 & 0.70 & 480 & & & & \\
\hline Mark 507 & 1.94 & 1205 & 1565 & 6 & 0.50 & 1025 & & & & \\
\hline HS $1817+5342$ & 0.59 & 1625 & 1615 & 79 & 0.20 & 1000 & 570 & 1215 & -375 & 2.21 \\
\hline HS $1831+5338$ & 0.74 & 1470 & 1555 & 32 & 0.30 & 240 & & & & \\
\hline Mark 896 & 0.50 & 1015 & 1135 & 32 & 0.19 & 315 & & & & \\
\hline MS $22102+1827$ & $<1.2$ & 820 & 690 & 42 & 0.16 & 890 & & & & \\
\hline Akn 564 & 0.67 & 710 & 865 & 48 & 0.92 & 220 & & & & \\
\hline HS $2247+1044$ & 1.11 & 1625 & 1790 & 30 & 0.12 & 710 & & & & \\
\hline Kaz 320 & 0.49 & 1160 & 1470 & 85 & 0.70 & 350 & 260 & 830 & -275 & 0.57 \\
\hline
\end{tabular}

Table 4. FWHM of the broad component of the $\mathrm{H} \alpha$ and $\mathrm{H} \beta$ lines when fitted either by a Gaussian or a Lorentzian profile. Column 1: name, Cols. 2 and 3: FWHM $\left(\mathrm{km} \mathrm{s}^{-1}\right)$ of the broad $\mathrm{H} \alpha$ and $\mathrm{H} \beta$ lines fitted with a Gaussian, Col. 5: ratio $(R)$ of the $F W H M$ of $\mathrm{H} \alpha$ to $\mathrm{H} \beta$, Cols. 6 and 7: FWHM $\left(\mathrm{km} \mathrm{s}^{-1}\right)$ of the broad $\mathrm{H} \alpha$ and $\mathrm{H} \beta$ lines fitted with a Lorentzian, Col. 8: ratio of the $F W H M$ of $\mathrm{H} \alpha$ to $\mathrm{H} \beta$.

\begin{tabular}{|c|c|c|c|c|c|}
\hline \multirow[b]{2}{*}{ Name } & \multicolumn{2}{|c|}{ Gaussian fit } & \multicolumn{3}{|c|}{ Lorentzian fit } \\
\hline & $\begin{array}{l}\mathrm{H} \alpha \quad \mathrm{H} \beta \\
F W H M\end{array}$ & & $\begin{array}{l}\mathrm{H} \alpha \\
F W\end{array}$ & $\begin{array}{c}\mathrm{H} \beta \\
H M\end{array}$ & \\
\hline MS $12235+2522$ & $2100 \quad 1585$ & $\overline{1.32}$ & 705 & $\overline{800}$ & $\overline{0.88}$ \\
\hline PG $1244+026$ & 1625 & 1.72 & 820 & 740 & 1.11 \\
\hline PG $1448+273$ & $3000 \quad 1890$ & 1.59 & 915 & 1050 & 0.87 \\
\hline HS $2247+1044$ & $3410 \quad 2280$ & 1.50 & 1625 & 1790 & 0.91 \\
\hline
\end{tabular}

\subsection{The Fe II emission}

\subsubsection{Correlation between the strength of the Fe II emission and the width of the broad $\mathrm{H} \beta$ component}

Typical AGNs have $R_{4570} \sim 0.4$ with $\sim 90 \%$ of objects in the range 0.1 to 1 (Osterbrock 1977a; Bergeron \& Kunth 1984). Moderately strong Fe II emission $\left(R_{4570}>1\right)$

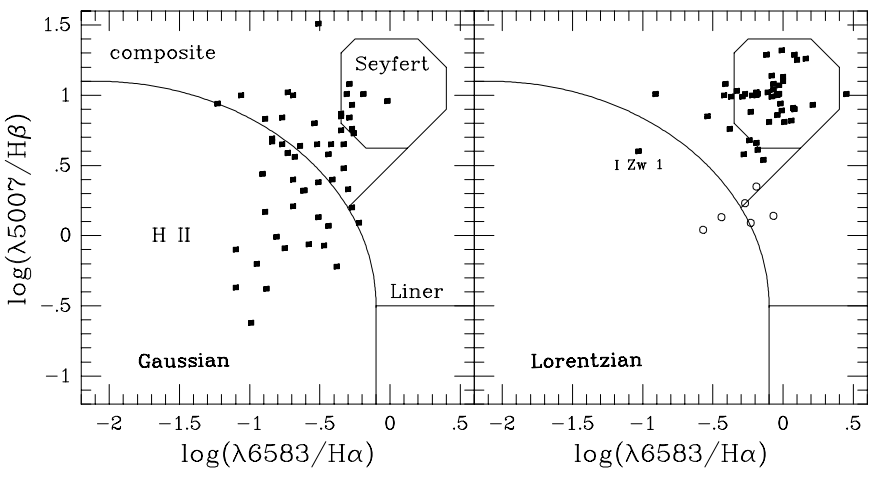

Fig. 8. Diagnostic diagrams for the narrow line region of the objects in our sample. In the left panel, the line ratios have been derived by fitting the broad Balmer components with a single Gaussian, while in the right panel we have used a single Lorentzian. In the right panel, Seyfert 2 s are shown by black squares and H II regions by open circles.

occurs in perhaps $5 \%$ of all objects (Lawrence et al. 1988). A few superstrong Fe II emitters $\left(R_{4570}>2\right)$ have been found (Lawrence et al. 1988; Lipari et al. 1993; Lipari 1994; Moran et al. 1996; Xia et al. 1999); they are listed in Table 5 . They are roughly an order of magnitude rarer. 


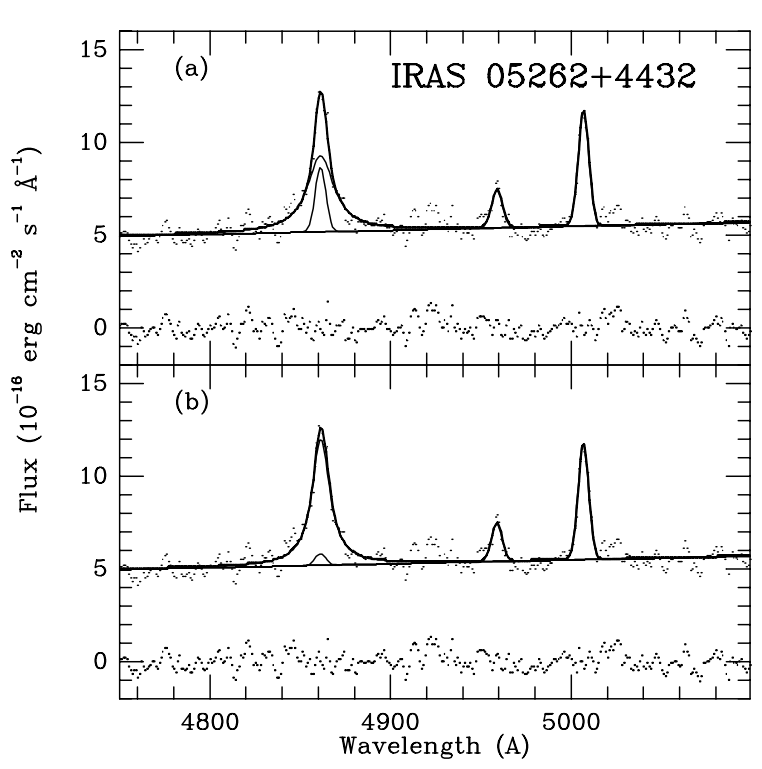

Fig. 9. In a), we have fitted the blue spectrum of IRAS $05262+4432$ with a set of Gaussian profiles for the narrow lines plus a single Lorentzian profile for the broad component of $\mathrm{H} \beta$; in $\mathbf{b}$ ), we forced the $\lambda 5007 / \mathrm{H} \beta$ ratio to be equal to 10. Examination of the residuals shows that the two solutions have equal merits.

Table 5. Known superstrong Fe II emitters $\left(R_{4570}>2\right)$.

\begin{tabular}{lccc}
\hline Name & Position & $R_{4570}$ & $\begin{array}{c}\mathrm{H} \beta_{\text {br }} F W H M \\
\mathrm{~km} \mathrm{~s}^{-1}\end{array}$ \\
\hline IRAS 04312+4008 & $0431+40$ & 2.4 & 1230 \\
IRAS 07598+6508 & $0759+65$ & 2.6 & 3200 \\
IRAS 10026+4347 & $1002+43$ & 2.0 & 2500 \\
IRAS 11598-0112 & $1159-01$ & 3.3 & 780 \\
Mark 231 & $1254+57$ & 2.1 & 3000 \\
IRAS 13224-3809 & $1322-38$ & 2.4 & 650 \\
Mark 507 & $1748+68$ & 2.9 & 965 \\
IRAS 18508-7815 & $1850-78$ & 2.4 & 3100 \\
IRAS 23410+0228 & $2341+02$ & 4.0 & 970 \\
\hline
\end{tabular}

Wills (1982) was the first to suggest that $R_{4570}$ is roughly inversely proportional to line width ( $F W H M$ of the broad $\mathrm{H} \beta$ component). Gaskell (1985) showed that $R_{4570}$ increases dramatically for $F W H M<1600 \mathrm{~km} \mathrm{~s}^{-1}$, but is relatively constant for $F W H M>1600 \mathrm{~km} \mathrm{~s}^{-1}$. Zheng \& Keel (1991) found that for AGNs with $F W H M>6000 \mathrm{~km} \mathrm{~s}^{-1}$, the mean value of $R_{4570}$ is 0.21 , less than half of that of the other objects, confirming that strong Fe II emission is not found in objects showing very broad emission lines; they showed that this is not an artifact resulting from blending of the Fe II lines when they are broad. Boroson \& Green (1992), followed by Wang et al. (1996) and Rodríguez-Ardila et al. (2000a), confirmed the existence of a strong anticorrelation between $R_{4570}$ and $\mathrm{H} \beta$ FWHM. This anticorrelation could be due

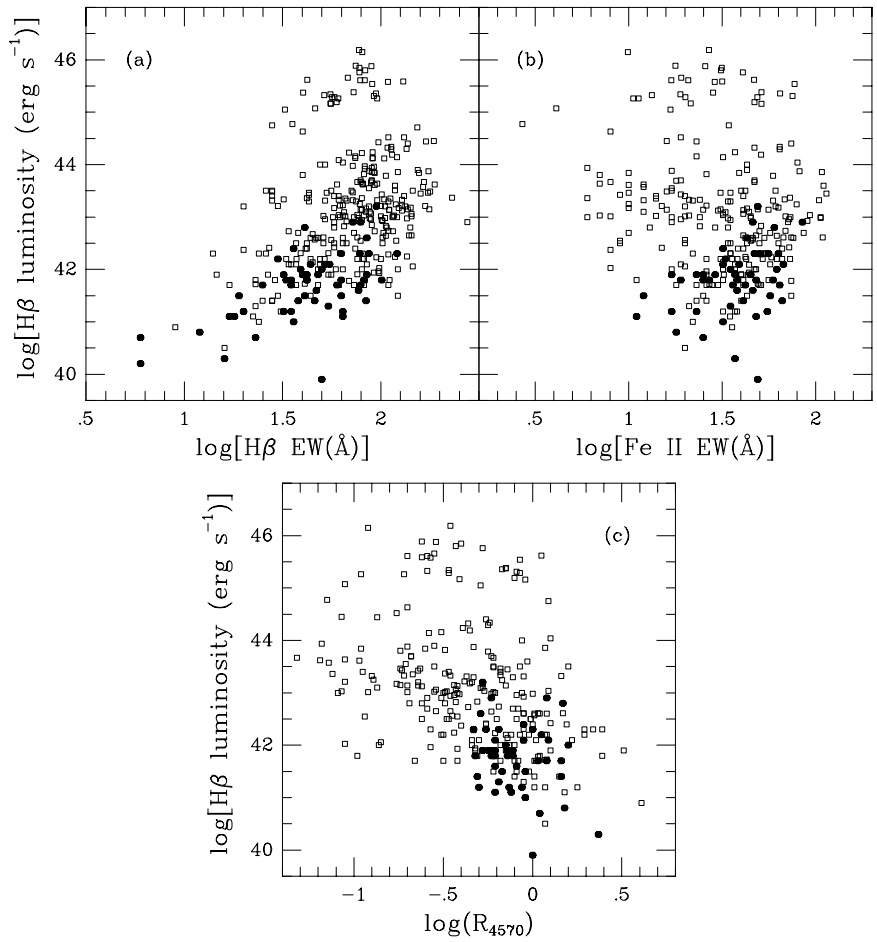

Fig. 10. Plots of the broad $\mathrm{H} \beta$ luminosity: in a) vs. the $E W$ of the broad $\mathrm{H} \beta$ component; the two parameters are highly correlated $(r=0.43)$ for low luminosity objects $\left(L(\mathrm{H} \beta)<10^{42.5} \mathrm{erg} \mathrm{s}^{-1}\right)$; but the $\mathrm{H} \beta E W$ s saturate at $\sim 100 \AA$ for larger luminosity objects; in b), vs. the Fe II $E W$; it is a scatter diagram $(r=0.20)$; in c) vs. $R_{4570}$. The anticorrelation observed $(r=0.42)$ is clearly due to the weakness of $\mathrm{H} \beta$ in the low luminosity objects rather than to the strength of $R_{4570}$. Several samples of Seyfert 1s have been used; our own is shown as filled circles; others (see text) are shown as open squares.

either to the existence of an anticorrelation between Fe II $E W$ and $\mathrm{H} \beta$ FWHM (Zheng \& O'Brien 1990; Boroson \& Green 1992) or of a correlation between $\mathrm{H} \beta E W$ and FWHM (Osterbrock 1977a; Gaskell 1985; Goodrich 1989).

We have plotted in Fig. 10 the broad $\mathrm{H} \beta$ luminosity vs. the $\mathrm{H} \beta_{\text {br }} E W$, the Fe II $E W$ and $R_{4570}$ for all objects in our sample and in those of Boroson \& Green (1992); Brotherton (1996); Moran et al. (1996); Marziani et al. (1996); Corbin \& Boroson (1996); Corbin (1997); Grupe et al. (1999) and McIntosh et al. (1999); Grupe et al. reported the $E W$ of the whole Fe II complex from 4250 to $5880 \AA$ A Leighly (1999b) used $30 \%$ of this value; we used $20 \%$. This figure clearly shows that the anticorrelation between $\mathrm{H} \beta$ luminosity and $R_{4570}(r=0.42)$ is the result of the correlation between $\mathrm{H} \beta$ luminosity and $E W(r=0.43)$ as there is no correlation between $\mathrm{H} \beta$ luminosity and Fe II $E W(r=0.20)$ (here and in what follows, $r$ is the linear correlation coefficient; the number of degrees of freedom is in each case $\sim 290$ ). This confirms Gaskell (1985) result that NLS1s have weak $\mathrm{H} \beta$ rather than strong Fe II. At very high densities $\left(N_{\mathrm{e}}>10^{10} \mathrm{~cm}^{-3}\right)$, the hydrogen lines become thermalized and their intensity drops considerably 
(Rees et al. 1989) which is a possible explanation of the decrease of the $\mathrm{H} \beta E W$ in NLS1s (Gaskell 1985).

\subsubsection{Correlation between the strength of the Fe II and [O III] emissions}

Goodrich (1989) noted that a defining characteristic of NLS1s is that the ratio $R_{5007}$ of the [O III] $\lambda 5007$ flux to the total $\mathrm{H} \beta$ flux is $<3$. In fact, when the first NLS1s were observed spectroscopically, the spectra were of relatively low resolution and the permitted and forbidden lines were believed to have the same width; these objects could therefore be mistaken for Seyfert $2 \mathrm{~s}$ except for the presence in their spectra of strong Fe II emission, a strong blue continuum and a small $\lambda 5007 / \mathrm{H} \beta$ ratio due to the unrecognized presence of a broad $\mathrm{H} \beta$ component; as, in Seyfert $2 \mathrm{~s}$, this ratio is larger than 3 , a smaller value was an indication of the presence of a broad $\mathrm{H} \beta$ component. But Osterbrock (1981) has divided the Seyfert 1s into five subgroups: S1.0, 1.2, 1.5, 1.8 and 1.9 on the basis of the appearance of the Balmer lines; a quantitative definition of these subgroups has been given by Winkler (1992) using the value of $R_{5007}$ : for S1.0s, $R_{5007}<0.2$, for S1.2, $0.2<R_{5007}<0.5$, for S1.5, $0.5<R_{5007}<3$. The higher values of $R_{5007}$ observed in S1.8 and S1.9 are believed to be due to partial extinction of the broad $\mathrm{H} \beta$ component. It follows that, for all S1s which do not suffer extinction, the condition $R_{5007}<3$ is fulfilled. We have measured on our spectra the parameter $R_{5007}$ (given in Table 3 ); we have excluded for the computation of $R_{5007}$ the contribution of the H II regions mentioned above. All objects have $R_{5007}$ values lower than 0.8 except IC 3599 for which it exceeds 3 .

Boroson \& Green (1992); Grupe et al. (1999) and McIntosh et al. (1999) found that Fe II is strong in objects with weak [O III] lines and vice versa. Let us note however that while Grupe et al. and McIntosh et al. found an anticorrelation between $R_{5007}$ and Fe II $E W$, Boroson $\&$ Green found a strong anticorrelation, not between the Fe II and $[\mathrm{O}$ III $] E W \mathrm{~s}$, but rather between the Fe II $E W$ and the ratio of the peak height of the $[\mathrm{O}$ III $] \lambda 5007$ line to that of $\mathrm{H} \beta$ which depends both on the value of $R_{5007}$ and the width of $\mathrm{H} \beta$; this correlation results from the anticorrelation between $R_{4570}$ and the $\mathrm{H} \beta F W H M$.

In Fig. 11 , we have plotted $R_{5007}$ vs. $R_{4570}$ and Fe II $E W$. There is a weak anticorrelation between Fe II $E W$ and $R_{5007}$, but not between $R_{4570}$ and $R_{5007}$. Therefore we do not confirm that Fe II is strong when the [O III] lines are weak.

Figure 12 shows the broad $\mathrm{H} \beta$ luminosity vs. the broad $\mathrm{H} \beta$ and $\lambda 5007 E W \mathrm{~s}$, and $R_{5007}$; the $\mathrm{H} \beta$ luminosities and $\lambda 5007 \mathrm{EW}$ s are not correlated; the weak anticorrelation between $\mathrm{H} \beta$ luminosity and $R_{5007}$ is due to the weakness of the $\mathrm{H} \beta E W$ in low luminosity objects, rather than to the strength of the $\lambda 5007 \mathrm{EW}$.

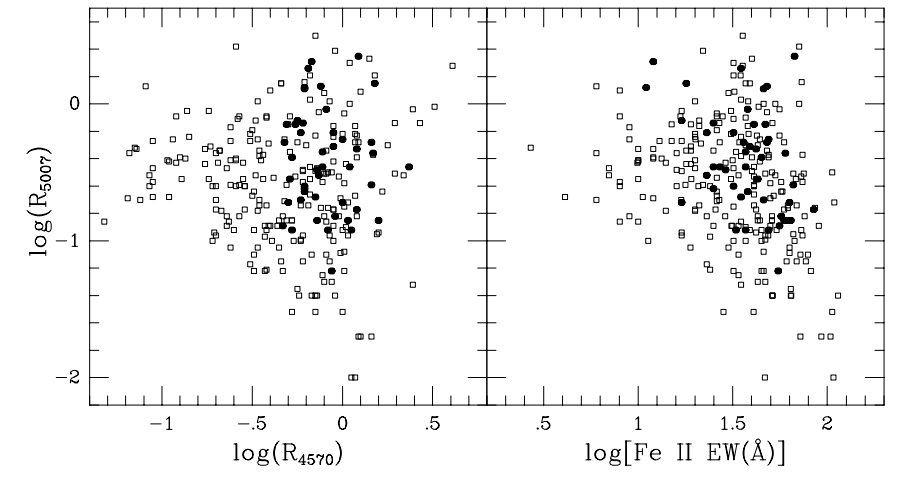

Fig. 11. Plot of $R_{5007}$ vs. $R_{4570}$ (left panel) and Fe II $E W$ (right panel) for several samples of Seyfert 1s. There is no correlation between $R_{5007}$ and $R_{4570}(r=0.05)$, while there is a trend for objects with a large Fe II $E W$ to have a weak $R_{5007}$ $(r=0.29)$. The symbols are as in Fig. 10 .
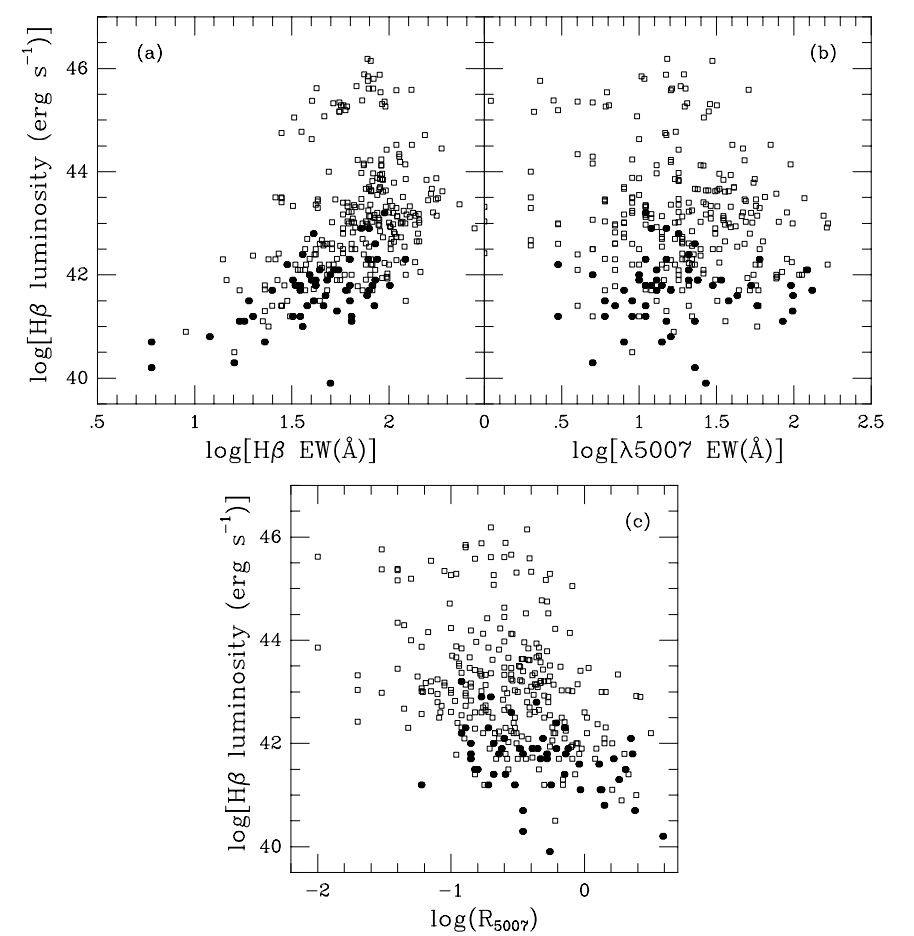

Fig. 12. Plots of the broad $\mathrm{H} \beta$ luminosity: in a) vs. the $E W$ of the broad $\mathrm{H} \beta$ component (as in Fig. 10); in b), vs. the $\lambda 5007 \mathrm{EW}$. It is a scatter diagram $(r=0.16)$. As a result, the $\mathrm{H} \beta$ luminosity is anticorrelated with $R_{5007}(r=0.43)$ c). The symbols are as in Fig. 10.

\subsection{On the definition of NLS1s}

Initially, as we have seen, NLS1s were defined as having narrow "broad" Balmer components $\left(<2000 \mathrm{~km} \mathrm{~s}^{-1}\right.$ $F W H M$ ). However, there is a continuous distribution of optical line widths in Seyfert 1s and the separation between BLS1s and NLS1s is arbitrary (Turner et al. 1999b; Sulentic et al. 2000a). Sulentic et al. (2000a) suggested that Seyfert 1s with $\mathrm{H} \beta F W H M<4000 \mathrm{~km} \mathrm{~s}^{-1}$ constitute a homogeneous class of objects having strikingly different line profiles compared to Seyfert 1s with broader lines. Grupe et al. (1999) have found objects displaying NLS1 properties (strong Fe II emission, a soft X-ray 
excess and variability) in spite of their $\mathrm{H} \beta F W H M$ exceeding $2000 \mathrm{~km} \mathrm{~s}^{-1}$. IRAS $10026+4347$ has a large soft X-ray excess $(\Gamma=3.2 \pm 0.5$ or $2.9 \pm 0.2)$, strong Fe II emission $\left(R_{4570}=2.0\right)$, and a high amplitude X-ray variability $(\times 8)$; but the $\mathrm{H} \beta F W H M$ is $\sim 2500$ or $2990 \mathrm{~km} \mathrm{~s}^{-1}$ (Grupe et al. 1998; Xia et al. 1999). Similarly, PDS 456 has a H $\beta$ $F W H M$ equal to $3500 \mathrm{~km} \mathrm{~s}^{-1}$ although the Fe II lines are relatively strong $\left(R_{4570}=0.46\right)$ (Simpson et al. 1999) and the X-ray spectrum shows a soft excess $(\Gamma=3.9 \pm 0.8)$ (Reeves et al. 2000; Vignali et al. 2000). A few objects are known which have strong Fe II emission and relatively broad $\mathrm{H} \beta$ lines, but no soft X-ray excess; Mark 231 and IRAS $07598+6508$ are two such examples. Mark 231 has a strong Fe II emission $\left(R_{4570}=2.03\right.$ or 1.60$)$, its $\mathrm{H} \beta$ FWHM is $\sim 3000 \mathrm{~km} \mathrm{~s}^{-1}$ (Boroson \& Meyers 1992; Lipari et al. 1993); the hard X-ray spectrum is heavily attenuated making it difficult to detect the eventual presence of a soft X-ray component (Turner 1999). IRAS 07598+6508 also has strong Fe II emission $\left(R_{4570}=2.60\right)$ and a relatively broad $\mathrm{H} \beta$ line $\left(2550-3200 \mathrm{~km} \mathrm{~s}^{-1} F W H M\right.$ ) (Lawrence et al. 1997; Lipari et al. 1993; Boroson \& Meyers 1992); it is probably a highly obscured X-ray source (Gallagher et al. 1999).

We have plotted in Fig. $13, \mathrm{H} \beta_{\mathrm{br}} F W H M$ vs. $R_{4570}$ for all objects in our sample as well as in the other samples listed above; this plot shows a trend for the objects with the strongest Fe II emission to have narrower Balmer lines, with $F W H M$ up to $3500 \mathrm{~km} \mathrm{~s}^{-1}$. We have drawn a line such that, for most objects below this line, $R_{4570}>0.50$ while, for many objects above it, $R_{4570}<0.50$. This could be a better definition of NLS1s.

Figure 14 is a plot of the ROSAT photon index $\Gamma$ vs. $R_{4570}$; open circles represent the objects above the line in Fig. 13 and filled circles the objects below this line. This figure shows a definite correlation between $\Gamma$ and $R_{4570}$. Although the objects with strong Fe II emission (black dots) show a large dispersion in $\Gamma$, most objects with small Fe II have $\Gamma<2.9$.

Among the objects classified as NLS1s, having both narrow "broad" Balmer components and a strong Fe II emission, two have a very small photon index: IRAS $09571+8435(\Gamma=1.39)$ and Mark $507(\Gamma=1.68)$. Mark 507 has an intrinsic neutral hydrogen column density $N_{\mathrm{H}}=27 \times 10^{20} \mathrm{~cm}^{-2}$ (see notes), this value being derived assuming that the intrinsic X-ray spectrum is a single power-law; it is quite possible that the column density is even higher and hides a soft X-ray component; this could also be true for IRAS $09571+8435$.

The $F W H M$ of the broad $\mathrm{H} \beta$ component has been found to increase with the $\mathrm{H} \beta$ luminosity (Miller et al. 1992). Figure 15 is a plot of the $\mathrm{H} \beta$ luminosity vs. the $\mathrm{H} \beta$ FWHM; the correlation between these two parameters is indeed quite strong; the linear correlation coefficient is $r=0.76$ for 294 data points, corresponding to a probability $p<0.01 \%$ for no correlation between the two parameters; luminous BLS1s tend to have broader Balmer lines (but we should keep in mind that the published $\mathrm{H} \beta$ $F W H M$ have not been measured in a uniform way and that

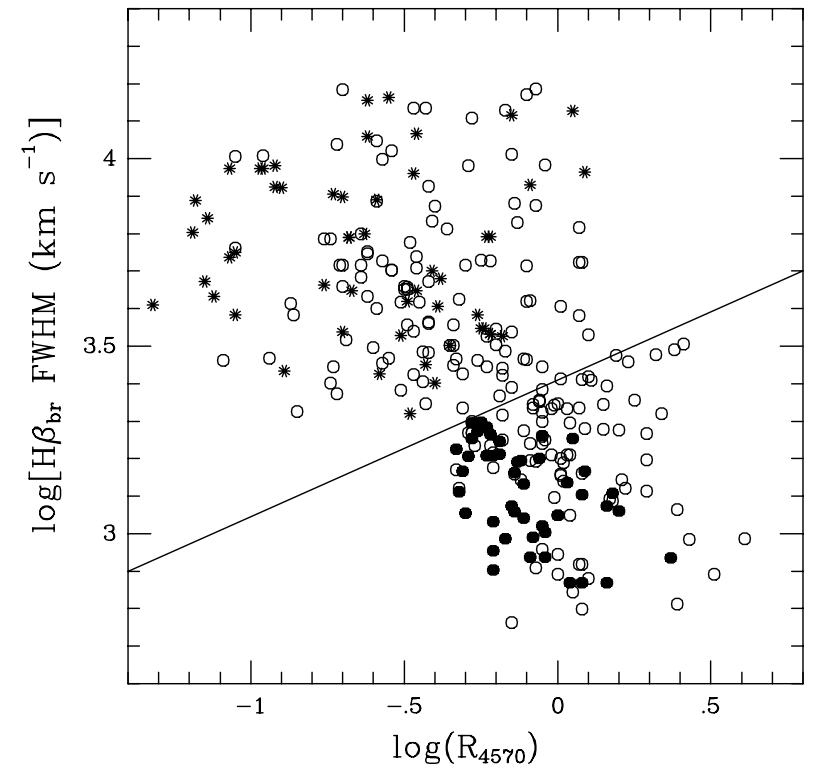

Fig. 13. Plot of the broad $\mathrm{H} \beta$ component $F W H M$ vs. $R_{4570}$. Filled circles represent the objects in our sample, open circles other radio quiet objects and stars radio loud objects; the correlation between the two parameters is strong $(r=0.54)$. The straight line divides the plane into BLS1s (above the line) and what could be the newly defined class of NLS1s (below the line).

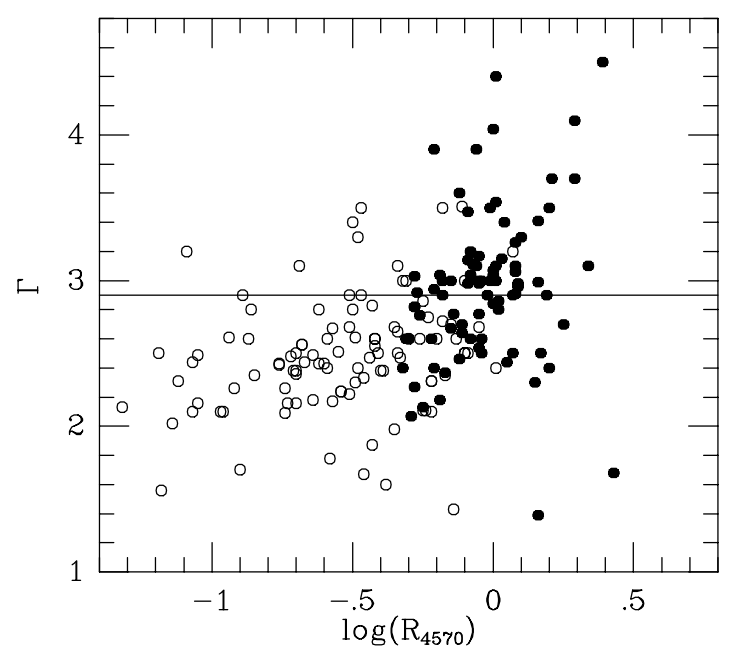

Fig. 14. Plot of the ROSAT photon index $\Gamma$ vs. $R_{4570}$. Filled circles represent the points below the line in the previous figure and open circles the points above the line. This diagram clearly shows that the soft X-ray excess tends to be large when the Fe II emission is large $(r=0.40)$.

the presence of the narrow component of the line has not always been taken properly into account). This leads to question whether the defining criterion for NLS1s should be a function of luminosity (Wills et al. 2000).

\section{Conclusions}

In most cases, the broad Balmer components of NLS1s are well fitted by a single Lorentzian profile, confirming previous claims that Lorentzian rather than Gaussian profiles 


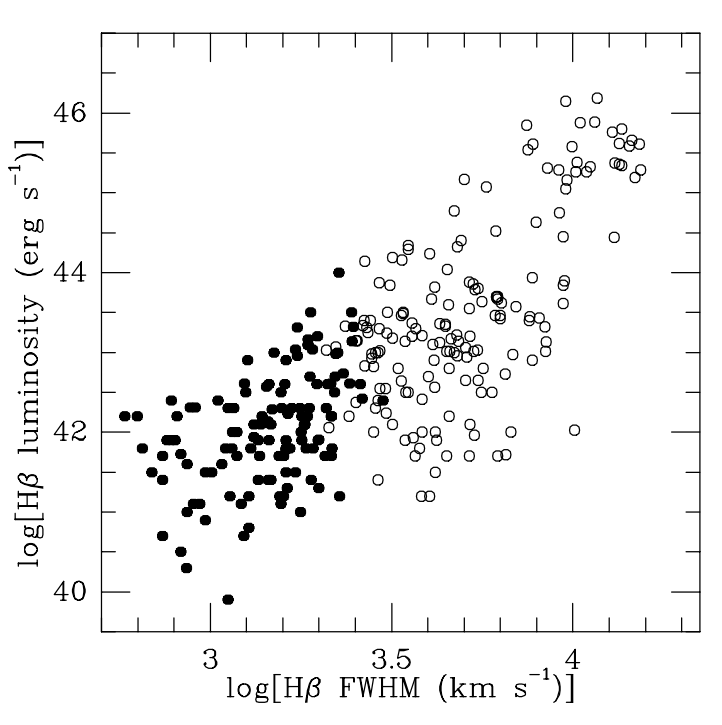

Fig. 15. Plot of the broad $\mathrm{H} \beta$ luminosity vs. the $F W H M$ of the broad $\mathrm{H} \beta$ component. The symbols are as in Fig. 14. There is clearly a strong correlation between the two plotted parameters $(r=0.76)$.

are better suited to reproduce the shape of the broad lines in NLS1s. This has consequences concerning their FWHMs and line ratios: when the broad Balmer components are fitted with a Lorentzian, most narrow line regions have line ratios typical of Seyfert $2 \mathrm{~s}$, while when they are fitted with a Gaussian they are widely scattered in the usual diagnostic diagrams; moreover, the $F W H M$ of the best fitting Lorentzian is systematically smaller than the $F W H M$ of the Gaussian. The Lorentzian shape of the broad emission lines could be the result of these lines being produced in a large disk.

We find that the [O III] lines usually have a relatively narrow Gaussian profile $\left(\sim 200-500 \mathrm{~km} \mathrm{~s}^{-1}\right.$ FWHM) with often, in addition, a second broad $\left(\sim 500-1800 \mathrm{~km} \mathrm{~s}^{-1}\right.$ FWHM), blueshifted Gaussian component. NLS1s.

We do not confirm that the [O III] lines are weak in

As previously suggested, there is a continuous transition of all properties between NLS1s and BLS1s. The strength of Fe II relative to $\mathrm{H} \beta\left(R_{4570}\right)$ could be the best parameter to recognize an NLS1 defined as a Seyfert 1 with a high accretion rate. The maximum FWHM of the broad Balmer component of NLS1s could be an increasing function of the $\mathrm{H} \beta$ luminosity.

As all objects with $\mathrm{H} \beta \quad F W H M$ smaller than $2000 \mathrm{~km} \mathrm{~s}^{-1}$ seem to be genuine NLS1s, we plan to make similar spectroscopic observations of a sizeable sample of Seyfert 1s with $2000<\mathrm{H} \beta F W H M<4000 \mathrm{~km} \mathrm{~s}^{-1}$ in the hope of better define the properties of those galaxies intermediate between NLS1s and BLS1s.

\section{Notes on individual objects}

Mark $335(0003+19)$ is an NLS1 with strong Fe II emission and narrow "broad" Balmer lines $\left(R_{4570}=0.62\right.$ and $\mathrm{H} \beta F W H M=1640 \mathrm{~km} \mathrm{~s}^{-1}$ ) (Boroson \& Green 1992) and relatively strong coronal lines (Grandi 1978).

The broad Balmer components clearly have a complex and asymmetrical profile (van Groningen 1987; Arav et al. 1997); to get a satisfactory fit of our spectra, two broad components were necessary, a Gaussian and a Lorentzian.

I Zw $1(0050+12)$ is the prototype NLS1; its optical spectrum reveals narrow emission lines and strong Fe II emission (Phillips 1976). The $\mathrm{H} \beta F W H M$ is in the range 1050-1500 $\mathrm{km} \mathrm{s}^{-1}$ (Osterbrock 1977a; Phillips 1978a; Peterson et al. 1982; de Robertis 1985) although Wilkes et al. (1999) measured $2590 \mathrm{~km} \mathrm{~s}^{-1}$. The broad Balmer components clearly have a complex and asymmetrical profile; to get a satisfactory fit of our spectra, two Lorentzians were needed.

An UV spectrum shows that ions of increasing ionization level show increasing excess blue-wing flux and an increasing line peak velocity shift (Laor et al. 1997b). UV absorption lines have been observed, indicating the likely presence of a warm absorber (Crenshaw et al. 1999).

Ton S180 (0054-22) is an NLS1 (Comastri et al. $1998)$ with strong Fe II emission $\left(R_{4570}=0.84\right.$; Comastri et al. 1998, or 1.02, Winkler 1992) and narrow Balmer lines $\left(F W H M \sim 1000 \mathrm{~km} \mathrm{~s}^{-1}\right.$ ) (Winkler 1992; Comastri et al. 1998; Grupe et al. 1999).

Mark $359(0124+18)$ is an NLS1 (Osterbrock \& Pogge 1985). The $F W H M$ of the broad $\mathrm{H} \alpha$ component is $780 \mathrm{~km} \mathrm{~s}^{-1}$; the forbidden lines are exceptionally narrow ( $F W H M \sim 100 \mathrm{~km} \mathrm{~s}^{-1}$ ) (Veilleux 1991a). Discrepent values have been published for $R_{4570}: 0.62$ (Goodrich 1989) and $<0.08$ (Osterbrock \& Pogge 1985). The coronal lines are very strong (Veilleux 1988; Erkens et al. 1997).

MS 01442-0055 (0144-00) is an NLS1 with $\mathrm{H} \beta$ $F W H M=1940 \mathrm{~km} \mathrm{~s}^{-1}$ and $R_{4570}=0.78$ (Stephens 1989).

Mark 1044 (0227-09) is an NLS1 with strong narrow Fe II emission (Osterbrock \& Dahari 1983). The $\mathrm{H} \beta$ FWHM is 1280 (Goodrich 1989) or $1400 \mathrm{~km} \mathrm{~s}^{-1}$ (Rafanelli 1985).

HS $\mathbf{0 3 2 8}+\mathbf{0 5 2 8}(0328+05)$. The spectra published by Perlman et al. (1996) and Engels et al. (1998) suggest that it is an NLS1, although they do not show the presence of Fe II emission. Our spectra show that the broad Balmer components are narrow $\left(\sim 1500 \mathrm{~km} \mathrm{~s}^{-1}\right)$ which confirms the classification as an NLS1.

The [N II] lines are extremely weak; we measured $\lambda 6583 / \mathrm{H} \alpha=0.12$.

IRAS $\mathbf{0 3 4 5 0 + 0 0 5 5}(0345+00)$. Boroson \& Meyers (1992) measured an $\mathrm{H} \alpha$ FWHM of $1310 \mathrm{~km} \mathrm{~s}^{-1}$ and $R_{4570}=0.96$. Giannuzzo \& Stirpe (1996) included it in their sample of NLS1s.

We have not observed this object.

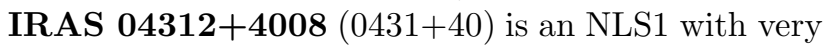
strong Fe II emission: $R_{4570}=2.36$ (Moran et al. 1996). 
It is located at low galactic latitude: $b=-5.0^{\circ}$ with $N_{\mathrm{H}} \sim$ $3.4 \times 10^{21} \mathrm{~cm}^{-2}$.

Mark 618 (0434-10). The Balmer line FWHM lies in the range 1760-2 $300 \mathrm{~km} \mathrm{~s}^{-1}$ (Osterbrock 1977a; Feldman \& MacAlpine 1978; Phillips 1978a; Peterson et al. 1982; Boroson \& Meyers 1992); it is a relatively strong Fe II emitter with $R_{4570}=0.50$ (Boroson \& Meyers 1992). It has however been classified as a Seyfert 1 rather than an NLS1 by Moran et al. (1996).

It is a X-ray source, variable on a time scale of $1000 \mathrm{~s}$ (Rao et al. 1992).

We have not observed this object.

IRAS $\mathbf{0 4 4 1 6 + 1 2 1 5}(0441+12)$ is an NLS1 with an $\mathrm{H} \beta F W H M=1670 \mathrm{~km} \mathrm{~s}^{-1}$ (Moran et al. 1996). The [O III] lines are very broad $\left(F W H M=1150 \mathrm{~km} \mathrm{~s}^{-1}\right.$ according to Moran et al. 1996); we find $1320 \mathrm{~km} \mathrm{~s}^{-1}$.

IRAS $\mathbf{0 4 5 7 6 + 0 9 1 2}(0457+09)$ is an NLS1 according to Moran et al. (1996) with $\mathrm{H} \beta F W H M=1220 \mathrm{~km} \mathrm{~s}^{-1}$ and $R_{4570}=1.51$. It is a $R O S A T$ X-ray source with a poorly determined photon index $\Gamma=1.4 \pm 1.0$ (Boller et al. 1992).

The $\left[\begin{array}{ll}\mathrm{O} & \mathrm{III}\end{array}\right]$ lines are very broad $(F W H M=$ $1310 \mathrm{~km} \mathrm{~s}^{-1}$ according to Moran et al. 1996; we find $\left.1290 \mathrm{~km} \mathrm{~s}^{-1}\right)$.

IRAS 04596-2257 (0459-22) is an NLS1 with $\mathrm{H} \beta$ $F W H M<1500 \mathrm{~km} \mathrm{~s}^{-1}$ (Green et al. 1989).

We have not observed this object.

IRAS $\mathbf{0 5 2 6 2 + 4 4 3 2}(0526+44)$ is an NLS1 with $\mathrm{H} \beta$ $F W H M=700 \mathrm{~km} \mathrm{~s}^{-1}$ (Moran et al. 1996).

The narrow lines are clearly extended on our spectra, with $\lambda 6583 / \mathrm{H} \alpha \sim 0.4$ outside the nucleus; the $33 \AA \mathrm{mm}^{-1}$ red nuclear spectrum is well fitted by a Lorentzian $\left(695 \mathrm{~km} \mathrm{~s}^{-1} F W H M\right)$ and two sets of narrow components with the same $\lambda 6583 / \mathrm{H} \alpha$ ratio $\sim 0.4$ clearly coming from a $\mathrm{H}$ II region. The blue narrow line spectrum is composite: a H II region with weak [O III] emission and a Seyfert 2 nebulosity with weak $\mathrm{H} \beta$ which is too weak to be detected in either $\mathrm{H} \alpha$ nor [N II].

It is a $R O S A T \mathrm{X}$-ray source with $\Gamma=5.0$, but the uncertainty in this value is large because of the high column density (Boller et al. 1992). It is located at a low galactic latitude: $b=5.7^{\circ}$ with $N_{\mathrm{H}} \sim 3.8 \times 10^{21} \mathrm{~cm}^{-2}$.

RX J07527+2617 $(0749+26)$ is an NLS1; the Balmer line $F W H M$ is $\sim 1000 \mathrm{~km} \mathrm{~s}^{-1}$ (Bade et al. 1995).

Mark $382(0752+39)$ is an NLS1; the $\mathrm{H} \beta F W H M$ is 1500 (Osterbrock 1977a) or $1400 \mathrm{~km} \mathrm{~s}^{-1}$ (Peterson et al. 1982). We measured $1280 \mathrm{~km} \mathrm{~s}^{-1}$.

Mark $110(0921+52)$ is a Seyfert 1 (Hutchings \& Craven 1988). The $\mathrm{H} \beta \quad F W H M$ is in the range 1670-2500 km s${ }^{-1}$ (Osterbrock 1977a; Crenshaw 1986; Boroson \& Green 1992; Bischoff \& Kollatschny 1999). The Fe II emission is weak $\left(R_{4570}=0.09-0.16\right)$ (Osterbrock 1977a; Meyers \& Peterson 1985; Boroson \& Green 1992); the Fe II line flux remains constant while the Balmer line fluxes vary (Bischoff \& Kollatschny 1999).

We have not observed this object.
Mark $705(0923+12)$. The $\mathrm{H} \beta F W H M$ is in the range 1670-2400 km s${ }^{-1}$ (Zheng \& O'Brien 1990; Miller et al. 1992; Boroson \& Green 1992). We found that the broad Balmer components have a $F W H M$ of $\sim 1770 \mathrm{~km} \mathrm{~s}^{-1}$. Coronal lines have been observed by Erkens et al. (1997).

Mark $707(0934+01)$ is an NLS1; the $\mathrm{H} \beta F W H M$ is 1320 (Boroson \& Green 1992) or $2000 \mathrm{~km} \mathrm{~s}^{-1}$ (Miller et al. 1992).

Mark $124(0945+50)$ is an NLS1 (De Grijp et al. 1992); the $F W H M$ of the broad $\mathrm{H} \beta$ component is in the range 1050-1400 $\mathrm{km} \mathrm{s}^{-1}$ (Osterbrock 1977a; Phillips 1978a). Grandi (1978) did not find high excitation lines.

Mark 1239 (0949-01) is an NLS1 (Osterbrock \& Pogge 1985); the FWHM of the broad Balmer components is $\sim 1000 \mathrm{~km} \mathrm{~s}^{-1}$ (Osterbrock \& Pogge 1985; de Robertis \& Osterbrock 1984). In contrast, Rodríguez-Ardila et al. (2000b) gave 2968 and $2278 \mathrm{~km} \mathrm{~s}^{-1} F W H M$ for the broad $\mathrm{H} \beta$ and $\mathrm{H} \alpha$ component respectively but called it however an NLS1, while Rafanelli \& Bonoli (1984) measured 5000 and $4800 \mathrm{~km} \mathrm{~s}^{-1}$ respectively. We have measured 1075 and $905 \mathrm{~km} \mathrm{~s}^{-1}$ respectively, confirming that the object is an NLS1. Coronal lines have been observed by Rafanelli \& Bonoli (1984) and Erkens et al. (1997).

IRAS $09571+8435(0957+84)$ is an NLS1 with $\mathrm{H} \beta$ $F W H M=1120 \mathrm{~km} \mathrm{~s}^{-1}$ and $R_{4570}=1.46$ (Moran et al. 1996).

Boller et al. (1992) found that the ROSAT photon index is $\Gamma=1.39$, a very small value for an NLS1, but the uncertainty is large.

PG 1011-040 (1011-04). The $\mathrm{H} \beta \quad F W H M$ is 1440 (Boroson \& Green 1992) or $1980 \mathrm{~km} \mathrm{~s}^{-1}$ (Miller et al. 1992). The spectrum published by Simpson et al. (1996) suggests that it is indeed an NLS1.

PG 1011-040 has been detected by $A S C A$ as an X-ray source with a photon index $\Gamma=1.93 \pm 0.41$; its $2-10 \mathrm{keV}$ luminosity is $6.3 \times 10^{41} \mathrm{erg} \mathrm{s}^{-1}$; it is a weak X-ray source for its optical luminosity (Gallagher et al. 2001).

PG 1016+336 $(1016+33)$ is an NLS1 (Osterbrock \& Pogge 1987). The $\mathrm{H} \beta F W H M$ is $1310 \mathrm{~km} \mathrm{~s}^{-1}$ and $R_{4570}=$ 0.87 (Goodrich 1989).

Mark $142(1022+51)$ is an NLS1; the $\mathrm{H} \beta F W H M$ is in the range 1350-1790 $\mathrm{km} \mathrm{s}^{-1}$ (Osterbrock 1977a; Phillips 1978a; Boroson \& Green 1992; Grupe et al. 1999).

KUG 1031+398 $(1031+39)$. The broad component of the Balmer lines is relatively narrow and, consequently, this object has been classified as an NLS1 by Puchnarewicz et al. (1995). The profile of the emission lines in KUG $1031+398$ is complex; four emission components are present: an extended H II region, two distinct Seyfert-type clouds identified with the NLR (one of which has quite broad lines: $\left.\sim 1115 \mathrm{~km} \mathrm{~s}^{-1} F W H M\right)$, and a relatively narrow "broad line" component $\left(1060 \mathrm{~km} \mathrm{~s}^{-1}\right.$ FWHM) (Gonçalves et al. 1999b).

According to Pounds et al. (1995), this object is unlike some other steep-spectrum (soft X-ray) AGNs in showing a marked absence of rapid X-ray variability and of strong Fe II line emission. 
RX J10407+3300 $(1037+33)$. The $F W H M$ of the Balmer lines is $1700 \mathrm{~km} \mathrm{~s}^{-1}$ and $R_{4570}=0.56$ (Bade et al. 1995) suggesting that it is an NLS1.

Mark $734(1119+12)$. The spectrum published by Simpson et al. (1996) suggests that it is an NLS1; the $\mathrm{H} \beta$ FWHM is 1820 (Boroson \& Green 1992) or $1940 \mathrm{~km} \mathrm{~s}^{-1}$ (Miller et al. 1992).

Mark 739E $(1133+21)$. The double nucleus nature of Mark 739 was first described by Petrosian et al. (1979). The eastern component is an NLS1 with $\mathrm{H} \alpha F W H M=$ $900 \mathrm{~km} \mathrm{~s}^{-1}$ and very strong Fe II emission (Netzer et al. 1987; Mazzarella \& Boroson 1993). Our Lorentzian fit to the Balmer lines has a $F W H M$ of $\sim 1500 \mathrm{~km} \mathrm{~s}^{-1}$, substantially larger than the published value.

MCG 06.26.012 $(1136+34)$ is an NLS1; the $\mathrm{H} \beta$ $F W H M$ is equal to $1685 \mathrm{~km} \mathrm{~s}^{-1}$ (Grupe et al. 1999). Our observations confirm this classification.

Mark $42(1151+46)$ is an NLS1 with relatively strong Fe II emission (Osterbrock \& Pogge 1985). The $\mathrm{H} \beta$ emission line is very narrow, in the range 550$670 \mathrm{~km} \mathrm{~s}^{-1}$ FWHM (Osterbrock \& Pogge 1985; Phillips 1978a). Grandi (1978) detected [Fe VII] 66087.

On our spectra, the narrow lines are very narrow $\left(<200 \mathrm{~km} \mathrm{~s}^{-1} F W H M\right.$ corrected for the instrumental broadening) and the line ratios are those of a H II region.

NGC $4051(1200+44)$ is an NLS1 (Leighly 1999a). The $\mathrm{H} \beta F W H M$ is 990 (De Robertis \& Osterbrock 1984) or $1150 \mathrm{~km} \mathrm{~s}^{-1}$ (Leighly 1999b). The coronal lines are strong (Grandi 1978; Veilleux 1988; Erkens et al. 1997). Peterson et al. (2000) found that the Balmer lines could arise in a disk-like configuration and the high-ionization lines in an outflowing wind, of which we observe preferentially the near side. The structure and the kinematics of the [O III] lines also suggest an outflow (Christopoulou et al. 1997).

Our observations show the presence of two components in the [O III] lines, a narrow one $\left(200 \mathrm{~km} \mathrm{~s}^{-1}\right.$ FWHM $)$ and a broader one $\left(665 \mathrm{~km} \mathrm{~s}^{-1} F W H M\right)$ blueshifted by $175 \mathrm{~km} \mathrm{~s}^{-1}$ with respect to the first.

The X-ray source is variable by at least a factor 30 (Papadakis \& Lawrence 1995; Leighly 1999a; Uttley et al. 1999; Komossa \& Meerschweinchen 2000); long term variations in the average X-ray flux might in principle be caused by absorption by a varying column of material along the line of sight; but this is ruled out by the spectral data; it is the $2-10 \mathrm{keV}$ luminosity which shows large amplitude long-term variations (Uttley et al. 1999).

PG 1211+143 $(1211+14)$. The broad Balmer component $F W H M$ has been measured to be in the range 1500-1860 (Zheng \& O'Brien 1990; Stirpe 1990, 1991; Appenzeller \& Wagner 1991; Miller et al. 1992; Boroson \& Green 1992; Wilkes et al. 1999), except for Miller et al. (1992) who found $2280 \mathrm{~km} \mathrm{~s}^{-1}$ for the $F W H M$ of $\mathrm{H} \alpha$. The [Fe VII] line was detected by Appenzeller \& Wagner (1991).

The soft $(0.1-2 \mathrm{keV}) \mathrm{X}$-ray flux varied by at least a factor of 16 (Yaqoob et al. 1994).
Mark $766(1215+30)$ is an NLS1 (Osterbrock \& Pogge 1985). The $\mathrm{H} \beta F W H M$ is 1600 (González Delgado \& Pérez 1996) or $2400 \mathrm{~km} \mathrm{~s}^{-1}$ (Osterbrock \& Pogge 1985). Our own measurements give 1150 and $1630 \mathrm{~km} \mathrm{~s}^{-1}$ for the broad $\mathrm{H} \alpha$ and $\mathrm{H} \beta$ components respectively. The spectrum shows relatively strong Fe II emission (Meyers \& Peterson 1985; González Delgado \& Pérez 1996) and coronal lines (Veilleux 1988; González Delgado \& Pérez 1996). The nucleus shows circumnuclear emission, the spectrum of which is well fitted by H II region models (González Delgado \& Pérez 1996).

MS 12170+0700 (1216+07) has been identified with an AGN (Maccacaro et al. 1994). Our spectra show strong broad Balmer components with FWHM equal to 1405 and $1765 \mathrm{~km} \mathrm{~s}^{-1}$ for $\mathrm{H} \alpha$ and $\mathrm{H} \beta$ respectively; the narrow line system is most probably a $\mathrm{H}$ II region as shown by the line ratios $(\lambda 5007 / \mathrm{H} \beta=1.2$ and $\lambda 6583 / \mathrm{H} \alpha=0.60)$.

MS $12235+2522(1223+25)$ is an NLS1 with $\mathrm{H} \beta$ $F W H M=1730 \mathrm{~km} \mathrm{~s}^{-1}$ and $\lambda 5007 F W H M=$ $1700 \mathrm{~km} \mathrm{~s}^{-1}$ according to Stephens (1989). The broad Balmer components in our spectra were both fitted by a single Lorentzian $\left(F W H M \sim 750 \mathrm{~km} \mathrm{~s}^{-1}\right)$.

IC $3599(1235+26)$. An optical spectrum, taken in May 1991, shows permitted lines with widths $\sim 1200$ $1500 \mathrm{~km} \mathrm{~s}^{-1}$ showing that this object is an NLS1; the forbidden lines are narrow and weak $\left(R_{5007}<0.1\right)$ (Brandt et al. 1995; Mason et al. 1995). Low dispersion spectra taken by Grupe et al. $(1995,1999)$ from 1992 to 1995 show narrow Balmer and forbidden lines placing this object close to the borderline between Seyfert 2 and H II galaxies in the diagnostic diagrams of Veilleux \& Osterbrock (1987); the resolution used was unsufficient to clearly show the composite nature of the spectrum; during this period, the line ratio $R_{5007}$ was constant $(3.3 \pm 0.3)$. Assuming that the $[\mathrm{O}$ III $] \lambda 5007$ line flux is not variable, it follows that, between May 1991 and February 1992, the flux of the Balmer lines has decreased by a factor $\sim 27$ (Grupe et al. 1995). [Fe VII] $\lambda 6087$ have been detected (Komossa \& Bade 1999).

Our spectra were taken in March 1997; the line ratio $R_{5007}$ was then $\sim 3.3$. The $\mathrm{H} \beta$ line was too weak for a significant fit to be made, but the broad $\mathrm{H} \alpha$ component could be fitted with a Lorentzian profile with a FWHM of $\sim 500 \mathrm{~km} \mathrm{~s}^{-1}$.

IC 3599 has been detected as a ROSAT X-ray source (Bade et al. 1995). The $0.1-2.5 \mathrm{keV}$ X-ray spectrum is extremely steep and is variable by an extremely large amount; the count-rate decreased by a factor of $\sim 80$ from December 1990 to June 1992 and then by an additional factor of $\sim 2$ to June 1993 (Grupe et al. 1995; Brandt et al. 1995; Mason et al. 1995).

PG 1244+026 (1244+02) is an NLS1; the FWHM of the $\mathrm{H} \beta$ line is 830 (Boroson \& Green 1992) or $1350 \mathrm{~km} \mathrm{~s}^{-1}$ (Miller et al. 1992).

NGC $4748(1249-13)$ is an NLS1 with strong Fe II emission (Osterbrock \& de Robertis 1985; Moran et al. 1996). The $\mathrm{H} \beta F W H M$ is in the range $1100-1500 \mathrm{~km} \mathrm{~s}^{-1}$ (Osterbrock \& de Robertis 1985; Maza \& Ruiz 1989; 
Winkler 1992). Rodríguez-Ardila et al. (2000b) measured $\sim 2350 \mathrm{~km} \mathrm{~s}^{-1}$ for the $F W H M$ of the broad Balmer components. Our own measurements show that they have a $F W H M$ of $\sim 1500 \mathrm{~km} \mathrm{~s}^{-1}$.

Mark $783(1300+16)$ is an NLS1, however the Fe II emission is very weak: $R_{4570}<0.11$ (Osterbrock \& Pogge 1985).

R $14.01(1338-14)$ is an NLS1 with $\mathrm{H} \beta F W H M=$ $1790 \mathrm{~km} \mathrm{~s}^{-1}$ (Maza \& Ruiz 1989).

Mark 69 (1343+29). Osterbrock (1977a) noted that it is a Seyfert 1 with relatively narrow "broad" Balmer lines ( $\left.1500 \mathrm{~km} \mathrm{~s}^{-1} F W H M\right)$. The emission lines on our spectra are well fitted with a single Lorentzian for the broad Balmer component and one set of Gaussians for the narrow lines, with line ratios indicating a $\mathrm{H}$ II region $(\lambda 5007 / \mathrm{H} \beta=2.23$ and $\lambda 6583 / \mathrm{H} \alpha=0.67)$.

2E $\mathbf{1 3 4 6 + 2 6 4 6}(1346+26)$ is a Seyfert 1 with a relatively narrow "broad" $\mathrm{H} \beta$ component (Hill \& Oegerle 1993). Our red spectrum is equally well fitted with either a broad Gaussian $\left(F W H M=1680 \mathrm{~km} \mathrm{~s}^{-1}\right)$ or a broad Lorentzian $\left(F W H M=1235 \mathrm{~km} \mathrm{~s}^{-1}\right)$. On the blue spectrum, the broad $\mathrm{H} \beta$ component is weak and the measurement quite uncertain.

PG 1404+226 $(1404+22)$ is an NLS1 with a narrow $\mathrm{H} \beta$ line: 880 (Boroson \& Green 1992) or $1290 \mathrm{~km} \mathrm{~s}^{-1}$ FWHM (Miller et al. 1992).

The X-ray flux has changed by a factor 13.1 in 10 years (Forster \& Halpern 1996) and by a factor 4 in $\sim 3 \times 10^{4} \mathrm{~s}$ (Ulrich et al. 1999).

Mark $684(1428+28)$ is an NLS1 (Osterbrock \& Pogge 1987) with prominent Fe II emission (Persson 1988).

On the red spectrum, the broad line component is poorly fitted by a single Lorentzian; a second, Gaussian component is needed. The broad $\mathrm{H} \beta$ line is too weak for a meaningful fit to be made. The spectrum is composite in the sense that the narrow line region has two components, one with relatively broad lines, the other with very narrow lines and line ratios typical of a H II region.

Mark $478(1440+35)$ is an NLS1 (Gondhalekar et al. 1994; Moran et al. 1996). The $\mathrm{H} \beta F W H M$ is in the range 1300-1915 $\mathrm{km} \mathrm{s}^{-1}$ (Phillips 1978a; Peterson et al. 1982; Boroson \& Green 1992; Gondhalekar et al. 1994; Grupe et al. 1999). The Fe II emission is strong (Phillips 1977). Grandi (1978) could not detect high excitation lines.

PG 1448+273 $(1448+27)$ is an NLS1; the $\mathrm{H} \beta F W H M$ is in the range $910-1200 \mathrm{~km} \mathrm{~s}^{-1}$ (Stirpe 1991; Boroson \& Green 1992).

IRAS 15091-2107 (1509-21) is an NLS1 (Osterbrock \& de Robertis 1985), although Moran et al. (1996) rather classified it as a Seyfert 1. Goodrich (1989) measured $\mathrm{H} \beta F W H M=1480 \mathrm{~km} \mathrm{~s}^{-1}$ and $R_{4570}=0.54$. Winkler (1992) and Maza \& Ruiz (1989) measured 2000 and $1600 \mathrm{~km} \mathrm{~s}^{-1}$ respectively for the $\mathrm{H} \beta$ FWHM.

We have not observed this object.

MS 15198-0633 (1519-06) is an AGN (Margon et al. 1985). The $\mathrm{H} \beta \quad F W H M$ is equal to $1304 \mathrm{~km} \mathrm{~s}^{-1}$; the $[\mathrm{Fe}$ VII $] \lambda 6087$ line was not detected (Appenzeller \& Wagner 1991). We have only a red spectrum with a relatively poor signal-to-noise ratio.

Mark $486(1535+54)$. The $\mathrm{H} \beta F W H M$ is in the range 1410-1650 km s${ }^{-1}$ (Boroson \& Green 1992; de Robertis 1985; Osterbrock \& Shuder 1982; Boroson et al. 1985). The Fe II emission is relatively strong (Phillips 1978a). Erkens et al. (1997) have observed coronal lines.

The X-ray source has an $A S C A$ photon index $\Gamma=$ $2.02 \pm 0.93$ and a high neutral absorption column density $\left(N_{\mathrm{H}}=1.2 \times 10^{23} \mathrm{~cm}^{-2}\right.$ plus an unabsorbed component scattered by electrons towards the observer; its $2-10 \mathrm{keV}$ luminosity is $1.3 \times 10^{42} \mathrm{erg} \mathrm{s}^{-1}$; if the optical nucleus was also absorbed by such a large column density, it would not be observable and the galaxy would appear as a Seyfert 2 (Gallagher et al. 2001).

IRAS 15462-0450 (1546-04) has been identified with the northern 16.6 mag spiral member of a loose interacting pair (Strauss et al. 1992; Duc et al. 1997). It is an ultraluminous IR galaxy with a Seyfert 1 spectrum and strong Fe II lines (Duc et al. 1997); the [O III] lines are broad with $F W H M=1560 \mathrm{~km} \mathrm{~s}^{-1}$ (Kim et al. 1998).

On our spectra, the broad Balmer components have a $F W H M$ of $\sim 1700 \mathrm{~km} \mathrm{~s}^{-1}$; the narrow lines have a complex profile and can be fitted with two Gaussian systems: one has very narrow lines $\left(<180 \mathrm{~km} \mathrm{~s}^{-1} F W H M\right.$ corrected for instrumental broadening), with line ratios indicating that it is a $\mathrm{H}$ II region; the second system has very broad lines $\left(\sim 1600 \mathrm{~km} \mathrm{~s}^{-1} F W H M\right)$ and correspond to a Seyfert 2 nebulosity.

Mark $493(1557+35)$ is an NLS1 with $\mathrm{H} \beta F W H M=$ $410 \mathrm{~km} \mathrm{~s}^{-1}$ (Osterbrock \& Pogge 1985).

EXO $\mathbf{1 6 5 2 4}+\mathbf{3 9 3 0}(1652+39)$ is an NLS1 with Balmer line $F W H M$ s equal to $1000 \mathrm{~km} \mathrm{~s}^{-1}$ (Bassani et al. 1989), which is confirmed by our own observations.

B3 $\mathbf{1 7 0 2 + 4 5 7}(1702+45)$ is an NLS1 according to Moran et al. (1996) and Wisotzki \& Bade (1997) who give 490 and $800 \mathrm{~km} \mathrm{~s}^{-1}$ respectively for the $\mathrm{H} \beta F W H M$. Leighly (1999b) measured $R_{4570}=1.86$.

Komossa \& Bade (1998) have shown the presence of a warm absorber. The $A S C A$ spectrum is well fitted by a single power law $(\Gamma=2.20 \pm 0.06)$ plus Galactic absorption, a warm absorber and no soft excess (Leighly 1999b; Vaughan et al. 1999a).

RX J17450+4802 $(1743+48)$ is a Seyfert 1 (Perlman et al. 1996). The Balmer line $F W H M$ is $1600 \mathrm{~km} \mathrm{~s}^{-1}$ (Bade et al. 1995). Our blue spectrum shows that the $\mathrm{H} \beta$ broad component $F W H M$ is $1355 \mathrm{~km} \mathrm{~s}^{-1}$ and $R_{4570}=0.78$, so this object is an NLS1.

Kaz $163(1747+68)$ is the southern member of an interacting pair. It is an NLS1 (Stephens 1989). The $\mathrm{H} \beta F W H M$ is in the range $1040-2110 \mathrm{~km} \mathrm{~s}^{-1}$ (Kriss \& Canizares 1982; Stephens 1989; Goodrich 1989; Leighly 1999b).

Mark $507(1748+68)$ has been variously classified as a H II region (Terlevich et al. 1991), a Seyfert 2 (Koski 1978) 
or a Liner with a "transition" type nucleus (Heckman 1980). It is however an NLS1 according to Halpern \& Oke (1987); Goodrich (1989); Moran et al. (1996) and Leighly (1999b). The $\mathrm{H} \beta F W H M$ is 965 (Goodrich 1989) or $1150 \mathrm{~km} \mathrm{~s}^{-1}$ (Leighly 1999b). The Fe II emission is strong with $R_{4570}=2.71$ (Goodrich 1989) or 1.45 (Leighly 1999b).

Our spectra show narrow "broad" Balmer components $\left(\sim 1335 \mathrm{~km} \mathrm{~s}^{-1} F W H M\right)$. The [O III] lines and the narrow component of $\mathrm{H} \beta$ are best fitted by two sets of Gaussians, one with narrow components and weak [O III] lines, typical of a H II region, the other with much broader lines and a large $\lambda 5007 / \mathrm{H} \beta$ ratio. The presence of the $\mathrm{H}$ II region component is not very surprising as Halpern \& Oke (1987) have found that the emission lines away from the nucleus are similar to those of a H II region.

The ROSAT photon index is $\Gamma=1.68 \pm 0.16$ (Leighly $1999 b)$, with an intrinsic neutral hydrogen column density: $N_{\mathrm{H}}=27 \times 10^{20} \mathrm{~cm}^{-2}$ in excess of the Galactic value (Iwasawa et al. 1998; Leighly 1999b). The photon index of this object is very small for an NLS1; this could be due to the presence of a high column density.

HS $\mathbf{1 8 1 7}+\mathbf{5 3 4 2}(1817+53)$. A spectrum published by Engels et al. (1998) suggests that this object could be an NLS1. Our spectra show that the broad Balmer line component $F W H M$ are $\sim 1620 \mathrm{~km} \mathrm{~s}^{-1}$ and $R_{4570}=0.59$, so there is no doubt that this is an NLS1.

HS $\mathbf{1 8 3 1 + 5 3 3 8}(1831+53)$. A spectrum published by Engels et al. (1998) suggests that this object could be an NLS1. Our spectra show that the FWHMs of the broad Balmer components are $\sim 1510 \mathrm{~km} \mathrm{~s}^{-1}$ and $R_{4570}=0.74$, so there is no doubt that this is an NLS1.

Mark 896 (2043-02) is a Seyfert 1 with relatively narrow Balmer lines and strong Fe II emission (Osterbrock \& Dahari 1983; Morris \& Ward 1988). The $\mathrm{H} \beta$ FWHM is 1390 (Stirpe 1991) or $1330 \mathrm{~km} \mathrm{~s}^{-1}$ (Stirpe 1990). However Moran et al. (1996) classified it as a Seyfert 1 rather than an NLS1. Our spectra show quite narrow "broad" Balmer components $\left(\sim 1100 \mathrm{~km} \mathrm{~s}^{-1}\right)$ and $R_{4570}=0.50$, so it is an NLS1.

MS 22102+1827 $(2210+18)$ has been identified with an AGN (Stocke et al. 1991; Maccacaro et al. 1994). Our spectra show quite narrow "broad" Balmer components $\left(\sim 750 \mathrm{~km} \mathrm{~s}^{-1}\right)$, so this is most probably an NLS1.

Akn $564(2240+29)$ is an NLS1 (Goodrich 1989). The $\mathrm{H} \alpha$ and $\mathrm{H} \beta \quad F W H M$ lie in the ranges $600-730$ and $720-1030 \mathrm{~km} \mathrm{~s}^{-1}$ respectively (Osterbrock \& Shuder 1982; de Robertis \& Osterbrock 1984; Stirpe 1990, 1991; Moran et al. 1996; Comastri et al. 2001). The coronal lines are strong (Veilleux 1988; Erkens et al. 1997; Comastri et al. 2001).

The X-ray flux varies by $\sim 50 \%$ in $1.6 \mathrm{~h}$; the X-ray light-curve shows no evidence for energy dependence of the variability within the $0.6-10 \mathrm{keV}$ bandpass (Turner et al. 1999a; Vaughan et al. 1999b).

UV absorption lines have been detected, indicating the presence of a warm absorber (Crenshaw et al. 1999).
HS $\mathbf{2 2 4 7 + 1 0 4 4}(2247+10)$. A spectrum published by Engels et al. (1998) suggested that this object could be an NLS1. This classification is confirmed by our spectra.

Kaz $320(2257+24)$ is an NLS1 according to Zamorano et al. (1992) who measured 1700 and $1800 \mathrm{~km} \mathrm{~s}^{-1}$ for the $F W H M$ of the broad component of $\mathrm{H} \alpha$ and $\mathrm{H} \beta$ respectively. Our spectra confirm this classification.

Acknowledgements. We thank C. Boisson for a careful reading of the manuscript.

\section{References}

Aoki, K., \& Yoshida, M. 1999, ASP Conf. Ser., 162, 385

Appenzeller, I., \& Wagner, S. J. 1991, A\&A, 250, 57

Arav, N., Barlow, T. A., Laor, A., \& Blandford, R. D. 1997, MNRAS, 288, 1015

Arribas, S., \& Mediavilla, E. 1993, ApJ, 410, 552

Bade, N., Fink, H. H., Engels, D., et al. 1995, A\&AS, 110, 469

Bassani, L., Coe, M. J., Malkan, M. A., et al. 1989, ApJ, 344, 726

Bergeron, J., \& Kunth, D. 1984, MNRAS, 207, 263

Bischoff, K., \& Kollatschny, W. 1999, A\&A, 345, 49

Boller, T., Meurs, E. J. A., Brinkmann, W., et al. 1992, A\&A, 261, 57

Boller, T., Brandt, W. N., \& Fink, H. 1996, A\&A, 305, 53

Boroson, T. A., \& Green, R. F. 1992, ApJS, 80, 109

Boroson, T. A., \& Meyers, K. A. 1992, ApJ, 397, 442

Boroson, T. A., Persson, S. E., \& Oke, J. B. 1985, ApJ, 293, 120

Bottorff, M., Korista, K. T., Schlosman, I., \& Blandford, R. D. 1997, ApJ, 479, 200

Brandt, W. N., Pounds, K. A., \& Fink, H. 1995, MNRAS, 273, L47

Brandt, W. N., Mathur, S., \& Elvis, M. 1997, MNRAS, 285, L25

Brandt, W. N., Boller, T., Fabian, A. C., \& Ruszkowski, M. 1999, MNRAS, 303, L53

Brotherton, M. S. 1996, ApJS, 102, 1

Brotherton, M. S., Wills, B. J., Francis, P. J., \& Steidel, C. C. 1994, ApJ, 430, 495

Christopoulou, P. E., Holloway, A. J., Steffen, W., et al. 1997, MNRAS, 284, 385

Ciliegi, P., \& Maccacaro, T. 1996, MNRAS, 282, 477

Clavel, J., Joly, M., Collin-Souffrin, S., Bergeron, J., \& Penston, M. V. 1983, MNRAS, 202, 85

Colbert, E. J. M., Baum, S. A., Gallimore, J. F., et al. 1996, ApJS, 105, 75

Collin-Souffrin, S., \& Lasota, J.-P. 1988, PASP, 100, 1041

Collin-Souffrin, S., Dumont, S., Heidmann, N., \& Joly, M. 1980, A\&A, 83, 190

Collin-Souffrin, S., Dyson, J. E., McDowell, J. C., \& Perry, J. J. 1988a, MNRAS, 232, 539

Collin-Souffrin, S., Hameury, J.-M., \& Joly, M. 1988b, A\&A, 205, 19

Comastri, A., Fiore, F., Guainazzi, M., et al. 1998, A\&A, 333, 31

Comastri, A., Stirpe, G. M., Vignali, C., et al. 2001, A\&A, 365, 400

Corbin, M. R. 1995, ApJ, 447, 496

Corbin, M. R. 1997, ApJS, 113, 245 
Corbin, M. R., \& Boroson, T. A. 1996, ApJS, 107, 69

Crenshaw, D. M. 1986, ApJS, 62, 821

Crenshaw, D. M., \& Kraemer, S. B. 2000, ApJ, 532, L101

Crenshaw, D. M., Kraemer, S. B., Boggess, A., et al. 1999, ApJ, 516, 750

Crenshaw, D. M., Kraemer, S. B., Hutchings, J. B., et al. 2000, AJ, 120,1731

de Grijp, M. H. K., Keel, W. C., Miley, G. K., Goudfrooij, P., \& Lub, J. 1992, A\&AS, 96, 389

de Robertis, M. M. 1985, ApJ, 289, 67

de Robertis, M. M., \& Osterbrock, D. E. 1984, ApJ, 286, 171

Duc, P. A., Mirabel, I. F., \& Maza, J. 1997, A\&AS, 124, 533

Dumont, A.-M., \& Collin-Souffrin, S. 1990, A\&A, 229, 313

Dumont, A.-M., Collin-Souffrin, S., \& Nazarova, L. 1998, A\&A, 331,11

Elvis, M., Green, R. F., Bectold, J., \& Fabbiano, G. 1986, ApJ, 310,291

Emmering, R. T., Blandford, R. D., \& Shlosman, I. 1992, ApJ, 385,460

Engels, D., Hagen, H.-J., Cordis, L., et al. 1998, A\&AS, 128, 507

Erkens, U., Appenzeller, J., \& Wagner, S. 1997, A\&A, 323, 707

Feldman, F. R., \& MacAlpine, G. M. 1978, ApJ, 221, 486

Fiore, F., Laor, A., Elvis, M., Nicastro, F., \& Giallongo, E. 1998, ApJ, 503, 607

Forster, K., \& Halpern, J. P. 1996, ApJ, 468, 565

Francis, P. J., Hewett, P. C., Foltz, C. B., \& Chaffee, F. H. 1992, ApJ, 398, 476

Gallagher, S. C., Brandt, W. N., Sambruna, R. M., et al. 1999, ApJ, 519, 549

Gallagher, S. C., Brandt, W. N., Laor, A., et al. 2001, ApJ, 546,795

Gaskell, C. M. 1985, ApJ, 291, 112

Gaskell, C. M. 2000, New Astron. Rev., 44, 563

George, I. M., Turner, T. J., Netzer, H., et al. 1998a, ApJS, 114,73

George, I. M., Mushotzky, R., Turner, T. J., et al. 1998b, ApJ, 509,146

George, I. M., Turner, T. J., Yaqoob, T., et al. 2000, ApJ, 531, 52

Giannuzzo, M. E., \& Stirpe, G. M. 1996, A\&A, 314, 419

Gliozzi, M., Boller, T., Brinkmann, W., \& Brandt, W. N. 2000, A\&A, 356, L17

Goad, M. R., Koratkar, A. P., Kim-Quijano, J., et al. 1999, ApJ, 524, 707

Gonçalves, A. C., Véron, P., \& Véron-Cetty, M.-P. 1999a, A\&AS, 135, 437

Gonçalves, A. C., Véron, P., \& Véron-Cetty, M.-P. 1999b, A\&A, 341, 662

Gondhalekar, P. M., Kellett, B. J., Pounds, K. A., Matthews, L., \& Quenby, J. J. 1994, MNRAS, 268, 973

González Delgado, R. M., \& Pérez, E. 1996, MNRAS, 278, 737

Goodrich, R. W. 1989, ApJ, 342, 224

Grandi, S. A. 1978, ApJ, 221, 501

Green, P. J., Ward, M. J., Anderson, S. F., et al. 1989, ApJ, 339, 93

Grupe, D., Beuerman, K., Mannheim, K., et al. 1995, A\&A, 299, L5

Grupe, D., Beuerman, K., Thomas, H. C., et al. 1998, A\&A, 330,25

Grupe, D., Beuerman, K., Mannheim, K., \& Thomas, H. C. 1999, A\&A, 350, 805

Halpern, J. P., \& Oke, J. B. 1987, ApJ, 312, 91 of IPC X-ray sources, Smithsonian astrophysical observatory, Cambridge
Heckman, T. M. 1980, A\&A, 87, 152

Heckman, T. M., Miley ,G. K., van Breugel, W. J. M., \& Butcher, H. R. 1981, ApJ, 247, 403

Heckman, T. M., Miley, G. K., \& Green, R. F. 1984, ApJ, 281, 525

Hill, J. M., \& Oegerle, W. R. 1993, AJ, 106, 831

Hutchings, J. B., \& Craven, S. E. 1988, AJ, 95, 677

Iwasawa, K., Brandt, W. N., \& Fabian, A. C. 1998, MNRAS, 293, 251

Joly, M. 1981, A\&A, 102, 321

Kaiser, M. E., Bradley, L. D., Hutchings, J. B., et al. 2000, ApJ, 528, 260

Kim, D.-C., Veilleux, S., \& Sanders, D. B. 1998, ApJ, 508, 627

Kolman, M., Halpern, J. P., Shrader, C. R., et al. 1993, ApJ, 402,514

Komossa, S., \& Fink, H. 1997a, A\&A, 327, 483

Komossa, S., \& Fink, H. 1997b, A\&A, 327, 555

Komossa, S., \& Bade, N. 1998, A\&A, 331, L49

Komossa, S., \& Bade, N. 1999, A\&A, 343, 775

Komossa, S., \& Meerschweinchen, J. 2000, A\&A, 354, 411

Königl, A., \& Kartje, J. F. 1994, ApJ, 434, 446

Koski, A. T. 1978, ApJ, 223, 56

Kriss, G. A., \& Canizares, C. R. 1982, ApJ, 261, 51

Kuraszkiewicz, J. K., Wilkes, B. J., Czerny, B., \& Mathur, S. 2000, ApJ, 542, 692

Kwan, J., Cheng, F.-Z., Fang, L.-Z., Zheng, W., \& Ge, J. 1995, ApJ, 440, 628

Laor, A., Fiore, F., Elvis, M., Wilkes, B. J., \& McDonnel, J. C. 1997a, ApJ, 477, 93

Laor, A., Jannuzi, B. T., Green, R. F., \& Boroson, T. A. 1997b, ApJ, 489, 656

Lawrence, A., Saunders, W., Rowan-Robinson, M., et al. 1988, MNRAS, 235, 261

Lawrence, A., Elvis, M., Wilkes, B. J., McHardy, I., \& Brandt, N. 1997, MNRAS, 285, 879

Leighly, K. M. 1999a, ApJS, 125, 297

Leighly, K. M. 1999b, ApJS, 125, 317

Leighly, K. M. 2001, HST SIS ultraviolet spectral evidence for outflows in extreme narrow-line Seyfert 1 galaxies, ed. B. M. Peterson, R. S. Polidan, \& R. W. Pogge, ASP Conf. Ser., 224, in press

Lemaître, G., Kohler, D., Lacroix, D., Meunier, J.-P., \& Vin, A. 1989, A\&A, 228, 546

Lipari, S. 1994, ApJ, 436, 102

Lipari, S., Terlevich, R., \& Macchetto, F. 1993, ApJ, 406, 451

Maccacaro, T., Wolter, A., McLean, B., et al. 1994, Astrophys. Lett. \& Comm., 29, 267

Margon, B., Downes, R. A., \& Chanan, G. A. 1985, ApJS, 59, 23

Marziani, P., Sulentic, J. W., Dultzin-Hacyan, D., Calvani, M., \& Moles, M. 1996, ApJS, 104, 37

Mason, K. O., Hassall, B. J. M., Bromage, G. E., et al. 1995, MNRAS, 274, 1194

Massey, P., Strobel, K., Barnes, J. V., \& Anderson, E. 1988, ApJ, 328, 315

Mathews, W. G., \& Wampler, E. J. 1985, PASP, 97, 966

Maza, J., \& Ruiz, M. T. 1989, ApJS, 69, 353

Mazzarella, J. M., \& Boroson, T. A. 1993, ApJS, 85, 27

McIntosh, D. H., Rieke, M. J., Rix, H.-W., Foltz, C. B., \& Weyman, R. J. 1999, ApJ, 514, 40

Meyers, K. A., \& Peterson, B. M. 1985, PASP, 97, 734

Miller, P., Rawlings, S., Saunders, R., \& Eales, S. 1992, MNRAS, 254, 93 
Molthagen, K., Bade, N., \& Wendker, H. J. 1998, A\&A, 331, 925

Moran, E. C., Halpern, J. P., \& Helfand, D. J. 1996, ApJS, 106, 341

Morris, S. L., \& Ward, M. J. 1988, MNRAS, 230, 639

Murray, N., \& Chiang, J. 1998, ApJ, 494, 125

Nagao, T., Murayama, T., \& Taniguchi, Y. 2001, ApJ, 546, 744

Nandra, K., \& Pounds, K. A. 1994, MNRAS, 268, 405

Netzer, H., Kollatschny, W., \& Fricke, K. J. 1987, A\&A, 171, 41

Nicastro, F. 2000, ApJ, 530, L65

Oke, J. B. 1974, ApJS, 27, 21

Oke, J. B., \& Gunn, J. E. 1983, ApJ, 206, 713

Osterbrock, D. E. 1977a, ApJ, 215, 733

Osterbrock, D. E. 1977b, PASP, 89, 620

Osterbrock, D. E. 1981, ApJ, 249, 462

Osterbrock, D. E. 1987, Lecture Notes in Physics, 307, 1

Osterbrock, D. E., \& Dahari, O. 1983, ApJ, 273, 478

Osterbrock, D. E., \& Shuder, J. M. 1982, ApJS, 49, 149

Osterbrock, D. E., \& de Robertis, M. M. 1985, PASP, 97, 1129

Osterbrock, D. E., \& Pogge, R. W. 1985, ApJ, 297, 166

Osterbrock, D. E., \& Pogge, R. W. 1987, ApJ, 323, 108

Page, M. J., Carrera, F. J., Mittaz, J. P. D., \& Mason, K. O. 1999, MNRAS, 305, 775

Papadakis, I. E., \& Lawrence, A. 1995, MNRAS, 272, 161

Perlman, E. S., Stocke, J. T., Schachter, J. F., et al. 1996, ApJS, 104, 251

Persson, S. E. 1988, ApJ, 330, 751

Peterson, B. M., Foltz, C. G., Byard, P. L., \& Wagner, R. M. 1982, ApJS, 49, 469

Peterson, B. M., Pogge, R. W., \& Wanders, I. 1999, ASP Conf. Ser., 175, 41

Peterson, B. M., McHardy, I. M., Wilkes, B. J., et al. 2000, ApJ, 542, 161

Petrosian, A. R., Saakian, K. A., \& Khachikian, E. E. 1979, Astrophys., 15, 250

Pfefferkorn, F., Boller, T., \& Rafanelli, P. 2001, A\&A, 368, 797

Phillips, M. M. 1976, ApJ, 208, 37

Phillips, M. M. 1977, ApJ, 215, 746

Phillips, M. M. 1978a, ApJS, 38, 187

Phillips, M. M. 1978b, ApJ, 226, 736

Pogge, R. W. 1989, ApJ, 345, 730

Pounds, K. A., Stanger, V. J., Turner, T. J., King, A. R., \& Czerny, B. 1987, MNRAS, 224, 443

Pounds, K. A., Done, C., \& Osborne, J. P. 1995, MNRAS, 277, L5

Puchnarewicz, E. M., Mason, K. O., Córdova, F. A., et al. 1992, MNRAS, 256, 589

Puchnarewicz, E. M., Mason, K. O., Córdova, F. A., et al. 1994, MNRAS, 270, 663

Puchnarewicz, E. M., Mason, K. O., Siemiginowska, A., \& Pounds, K. 1995, MNRAS, 276, 20

Puchnarewicz, E. M., Mason, K. O., Carrera, F. J., et al. 1997, MNRAS, 291, 177

Rafanelli, P. 1985, A\&A, 146, 17

Rafanelli, P., \& Bonoli, C. 1984, A\&A, 131, 186

Rao, A. R., Singh, K. P., \& Vahia, M. N. 1992, MNRAS, 255, 197

Rees, M. J., Netzer, H., \& Ferland, G. J. 1989, ApJ, 347, 640

Reeves, J., \& Turner, M. J. L. 2000, MNRAS, 316, 234

Reeves, J., O'Brien, P. T., Vaughan, S., et al. 2000, MNRAS, 312, L17
Reynolds, C. S. 1997, MNRAS, 286, 513

Rodríguez-Ardila, A., Pastoriza, M. G., \& Donzelli, C. J. 2000a, ApJS, 126, 63

Rodríguez-Ardila, A., Binette, L., Pastoriza, M. G., \& Donzelli, C. J. 2000b, ApJ, 538, 581

Rodríguez-Pascual, P. M., Mas-Hesse, J. M., \& Santos-Lléo, M. 1997, A\&A, 327, 72

Ross, R. R., Fabian, A. C., \& Mineshige, S. 1992, MNRAS, 258, 189

Rush, B., Malkan, M. A., Fink, H. H., \& Voges, W. 1996, ApJ, 471, 190

Sanders, D. B., Phinney, E. S., Neugebauer, G., Soifer, B. T., \& Matthews, K. 1989, ApJ, 347, 29

Saxton, R. D., Turner, M. J. L., Williams, O. R., et al. 1993, MNRAS, 262, 63

Siemiginowska, A., Kuhn, O., Elvis, M., et al. 1995, ApJ, 454, 77

Simpson, C., Ward, M., Clements, D. L., \& Rawlings, S. 1996, MNRAS, 281, 509

Simpson, C., Ward, M., O'Brien, P., \& Reeves, J. 1999, MNRAS, 303, L23

Smith, S. J. 1993, ApJ, 411, 570

Stephens, S. A. 1989, AJ, 97, 10

Stirpe, G. M. 1990, A\&AS, 85, 1049

Stirpe, G. M. 1991, A\&A, 247, 3

Stocke, J. T., Morris, S. L., Gioia, I. M., et al. 1991, ApJS, 76, 813

Stone, R. P. S. 1977, ApJ, 218, 767

Storchi-Bergmann, T., Wilson, A. S., \& Baldwin, J. A. 1992, ApJ, 396, 45

Strauss, M. A., Huchra, J. P., Davis, M., et al. 1992, ApJS, 83, 29

Sulentic, J. W., Marziani, P., Dultzin-Hacyan, D., Calvani, M., \& Moles, M. 1995, ApJ, 445, L85

Sulentic, J. W., Zwitter, T., Marziani, P., \& Dultzin-Hacyan, D. 2000a, ApJ, 536, L5

Sulentic, J. W., Marziani, P., Zwitter, T., Dultzin-Hacyan, D., \& Calvani, M. 2000b, ApJ, 545, L15

Sun, W.-H., \& Malkan, M. A. 1989, ApJ, 346, 68

Terlevich, R., Melnick, J., Masegosa, J., Moles, M., \& Copetti, M. V. F. 1991, A\&AS, 91, 285

Turner, T. J. 1999, ApJ, 511, 142

Turner, T. J., George, I. M., \& Netzer, H. 1999a, ApJ, 526, 52

Turner, T. J., George, I. M., Nandra, K., \& Turcan, D. 1999b, ApJ, 524, 667

Ulrich-Demoulin, M.-H., \& Molendi, S. 1996, ApJ, 457, 77

Uttley, P., McHardy, I. M., Papadakis, I. E., Guainazzi, M., \& Fruscione, A. 1999, MNRAS, 307, L6

van Groningen, E. 1987, A\&A, 186, 103

Vaughan, S., Reeves, J., Warwick, R., \& Edelson, R. 1999a, MNRAS, 309, 113

Vaughan, S., Pounds, K. A., Reeves, J., Warwick, R., \& Edelson, R. 1999b, MNRAS, 308, L34

Veilleux, S. 1988, AJ, 95, 1695

Veilleux, S. 1991a, ApJ, 368, 158

Veilleux, S. 1991b, ApJS, 75, 383

Veilleux, S., \& Osterbrock, D. E. 1987, ApJS, 63, 295

Veilleux, S., Shopbell, P. L., \& Miller, S. T. 2001, AJ, 121, 198

Véron, P., \& Véron-Cetty, M. P. 1986, A\&A, 161, 145

Véron, P., Lindblad, P. O., Zuiderwijk, E. J., Véron-Cetty, M. P., \& Adam, G. 1980, A\&A, 87, 245

Véron, P., Véron-Cetty, M. P., \& Zuiderwijk, E. J. 1981a, A\&A, 102,116 
Véron, P., Véron-Cetty, M. P., Bergeron, J., \& Zuiderwijk, E. J. Wills, B. J., Laor, A., Brotherton, M. S., et al. 1999, ApJ, 515, 1981b, A\&A, 97, 71

Véron, M.-P. 1981, A\&A, 100, 12

Véron-Cetty, M.-P., Véron, P., \& Gonçalves, A. C. 2001, in preparation

Vignali, C., Comastri, A., Nicastro, F., et al. 2000, A\&A, 362, 69

Vrtilek, J. M. 1985, ApJ, 294, 121

Vrtilek, J. M., \& Carleton, N. P. 1985, ApJ, 294, 106

Walter, R., \& Fink, H. H. 1993, A\&A, 274, 105

Wang, T., Brinkmann, W., \& Bergeron, J. 1996, A\&A, 309, 81

Wang, T.-G., Lu, Y.-J., \& Zhou, Y.-Y. 1998, ApJ, 493, 1

Whittle, M. 1985a, MNRAS, 213, 1

Whittle, M. 1985b, MNRAS, 213, 33

Whittle, M. 1992, ApJ, 387, 109

Whittle, M., Pedlar, A., Meurs, E. J. A., et al. 1988, ApJ, 326, 125

Wilkes, B. J., Kuraszkiewicz, J., Green, P. J., Mathur, S., \& McDonnel, J. C. 1999, ApJ, 513, 76

Wills, B. J. 1982, IAU Symp. 97, 373 L53

Wills, B. J., Shang, Z., \& Yuan, J. M. 2000, New Astron. Rev., 44,511

Wilson, A. S. 1994, in Oxford astrophysics workshop on Evidence for the torus, ed. M. J. Ward, 55

Wilson, A. S., Braatz, J. A., Heckman, T. M., Krolik, J. H., \& Miley, G. K. 1993, ApJ, 419, L61

Winkler, H. 1992, MNRAS, 257, 677

Wisotzki, L., \& Bade, N. 1997, A\&A, 320, 395

Wisotzki, L., Dreizler, S., Engels, D., Fink, H.-H., \& Heber, U. 1995, A\&A, 297, L55

Xia, X.-Y., Mao, S., Wu, H., et al. 1999, A\&A, 341, L13

Yaqoob, T., Serlemitsos, P., Mushotzky, R., et al. 1994, PASJ, 46, L173

Yuan, W., Brinkmann, W., Siebert, J., \& Voges, W. 1998, A\&A, 330, 108

Zamorano, J., Gallego, J., Rego, M., Vitores, A. G., \& Gonzalez-Riesta, R. 1992, AJ, 104, 1000

Zheng, W., \& O'Brien, P. T. 1990, ApJ, 353, 433

Zheng, W., \& Keel, W. C. 1991, ApJ, 382, 121

Wills, B. J., Brotherton, M. S., Fang, D., Steidel, C. C., \& Sargent, W. L. W. 1993, ApJ, 415, 563 